Yves Félix · Jean-Claude Thomas · Micheline Vigué-Poirrier

\title{
Rational string topology
}

Received June 10, 2004 and in revised form July 28, 2006

\begin{abstract}
We use the computational power of rational homotopy theory to provide an explicit cochain model for the loop product and the string bracket of a simply connected closed manifold $M$. We prove that the loop homology of $M$ is isomorphic to the Hochschild cohomology of the cochain algebra $C^{*}(M)$ with coefficients in $C^{*}(M)$. Some explicit computations of the loop product and the string bracket are given.
\end{abstract}

Keywords. String homology, rational homotopy, Hochschild cohomology, free loop space, loop space homology

\section{Introduction}

Let $C_{*}(X)$ (respectively $\left.H_{*}(X)\right)$ be the singular chains (respectively the singular homology) of a space $X$ with coefficients in $\mathbb{Q}$. For simplicity we identify $H_{*}(X \times X)$ with $H_{*}(X) \otimes H_{*}(X)$ and the singular cohomology $H^{*}(X)$ with the graded dual of the homology, i.e. $H^{*}(X):=H\left(C^{*}(X)\right)=\left(H_{*}(X)\right)^{\vee}$.

Let $M$ be a simply connected closed oriented $m$-manifold and let $L M$ (respectively $M^{I}$ and $\Omega M$ ) be the space of free loops (respectively paths and based loops). M. Chas and D. Sullivan [6] have constructed a product, called the loop product

$$
H_{*}(L M) \otimes H_{*}(L M) \rightarrow H_{*-m}(L M), \quad x \otimes y \mapsto x \bullet y,
$$

so that $\mathbb{H}_{*}(L M):=H_{*+m}(L M)$ is a commutative graded algebra, and a morphism, called the I-homomorphism

$$
I: \mathbb{H}_{*}(L M) \rightarrow H_{*}(\Omega M)
$$

The authors are partly supported by INTAS program 03513251.

Y. Félix: Département de mathématique, Université Catholique de Louvain, 2, Chemin du Cyclotron, 1348 Louvain-La-Neuve, Belgium; e-mail: felix@math.ucl.ac.be

J.-C. Thomas: Département de mathématique, Faculté des Sciences, 2, Boulevard Lavoisier, 49045 Angers, France; e-mail: jean-claude.thomas@univ-angers.fr

M. Vigué-Poirrier: Département de mathématique, Institut Galilée, Université de Paris-Nord, 93430 Villetaneuse, France; e-mail: vigue@ math.univ-paris13.fr

Mathematics Subject Classification (2000): 55P35, 54N45, 55N33, 17A65, 81T30, 17B55 
which relates the loop product structure on $\mathbb{H}_{*}(L M)$ with the Pontryagin algebra $H_{*}(\Omega M)$. The loop product induces the string bracket on the $S^{1}$-equivariant homology of $L M, \mathcal{H}_{*}=H_{*+m}^{S^{1}}(L M)$,

$$
\mathcal{H}_{*} \otimes \mathcal{H}_{*} \rightarrow \mathcal{H}_{*-2}, \quad a \otimes b \mapsto[a, b],
$$

such that $(\mathcal{H},[-,-])$ is a graded Lie algebra of degree 2. Few things are known about this bracket. For surfaces of genus larger than zero, Chas and Sullivan recover formulae proved in the context of symplectic geometry.

The purpose of this paper is to provide explicit computational tools for the duals of these two operations in cohomology and for the $I$-homomorphism.

First we will describe the dual of the loop product (Theorem A) and the dual of the string bracket (Theorem B) in terms of Sullivan models, [21]. It follows from Theorem A that the loop product structure on $H_{*}(L M)$ is invariant under orientation preserving maps which are quasi-isomorphisms. In Theorem $C$, we adapt the technics of Theorem A to a special type of Sullivan models, the cochains of a differential graded Lie algebra with coefficients in a differential graded Lie module. The main point in this paper is Theorem $\mathrm{D}$ which allows us to describe the dual of the loop product in terms of chains (instead of cochains) of a differential graded Lie algebra. This translation is performed by means of the "cap-homomorphism". This homomorphism realizes, in some sense, the Poincaré duality at the level of free loop spaces. The other interest for considering the caphomomorphism is that it allows us, in Theorem E, to identify the loop product in $\mathbb{H}_{*}(L M)$ with the Gerstenhaber product on the Hochschild cohomology $H H^{*}\left(C^{*}(M) ; C^{*}(M)\right)$. Here $C^{*}(M)$ denotes the cochain algebra of singular cochains on $M$.

Theorem E. Let $M$ be a simply connected closed oriented manifold. There exists a natural isomorphism of graded algebras

$$
J: \mathbb{H}_{*}(L M) \stackrel{\cong}{\rightarrow} H H^{*}\left(C^{*}(M) ; C^{*}(M)\right) .
$$

Such an identification has been proved by Merkulov [19] for the field of real numbers using iterated path integrals. Finally, we show

Theorem F. Let $J$ be the map in Theorem E. There exists a canonical isomorphism of graded algebras, $\bar{J}$, making the diagram

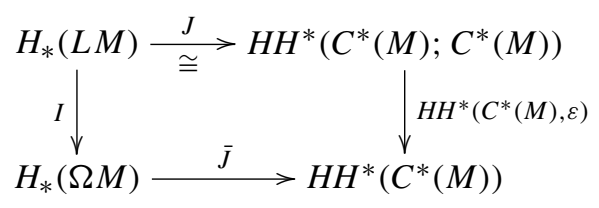

commutative, where $\varepsilon: C^{*}(M) \rightarrow \mathbb{Q}$ denotes the usual augmentation.

Theorems E and F are complementary results to our previous paper [14]. In particular, Theorems 2 and 3 in [14] apply verbatim. 
For the convenience of the reader we repeat the relevant material of rational homotopy theory, without proof, thus making our exposition self-contained. Moreover, we roughly indicate here the basic ideas of the paper.

Let $L$ be a graded differential Lie model of $M$. We denote by $U L_{a}$ its enveloping algebra considered as an $L$-module for the adjoint representation, and $U L_{a}^{\vee}$ its graded dual. Then the cochain algebra $\mathcal{C}^{*}\left(L ; U L_{a}^{\vee}\right)$ is a Sullivan model for the free loop space $L M$ and the map

$$
\mathcal{C}^{*}\left(L ; U L_{a}^{\vee}\right) \stackrel{\mathcal{C}^{*}\left(L ; \mu^{\vee}\right)}{\longrightarrow} \mathcal{C}^{*}\left(L ; U L_{a}^{\vee} \otimes U L_{a}^{\vee}\right)
$$

is a model for the composition of paths $L M \times_{M} L M \rightarrow L M$ where $\mu$ denotes the multiplication in $U L$.

On the other hand, if $(A \otimes \bigwedge W, d)$ is a (Sullivan) model of $L M$ with $A$ a finitedimensional model for $M$ satisfying Poincaré duality, there exists a linear map

$$
(A \otimes \wedge W, D) \otimes_{A}(A \otimes \bigwedge W, D) \rightarrow(A \otimes \wedge W, D)^{\otimes 2}
$$

which induces the cohomology Gysin map (see 4.5)

$$
i^{!}: H^{*}\left(L M \times{ }_{M} L M\right) \rightarrow H^{*+m}(L M \times L M)
$$

of the finite-codimensional embedding $i: L M \times{ }_{M} L M \hookrightarrow L M \times L M$. Since the dual of the loop product is the composition $H^{*}\left(c_{M}\right) \circ i^{!}$, the above constructions enable explicit calculations of the loop product.

The paper is organized as follows.

2. Preliminaries on differential homological algebra.

3. The dual of the loop product.

4. Theorem A. Dual of the loop product in terms of Sullivan models.

5. Theorem B. Dual of the string bracket in terms of Sullivan models.

6. Theorem C. Dual of the loop product in terms of cochains on a differential graded Lie algebra.

7. Theorem D. Dual of the loop product in terms of chains on a differential graded Lie algebra.

8. Theorem E. Loop product and Gerstenhaber product.

9. Theorem F. I-homomorphism and Hochschild homology.

\section{Preliminaries on differential homological algebra}

All the graded vector spaces, algebras, coalgebras and Lie algebras $V$ are defined over $\mathbb{Q}$ and are supposed to be of finite type, i.e. $\operatorname{dim} V_{n}<\infty$ for all $n$. 


\subsection{Graded vector spaces}

If $V=\left\{V_{i}\right\}_{i \in \mathbb{Z}}$ is a (lower) graded $\mathbb{Q}$-vector space (when we need upper graded vector space we put $V_{i}=V^{-i}$ as usual) then $V^{\vee}$ denotes the graded dual vector space and $s V$ denotes the suspension of $V$ :

$$
V^{\vee}=\operatorname{Hom}(V, \mathbb{Q}), \quad(s V)_{n}=V_{n-1}, \quad(s V)^{n}=V^{n+1} .
$$

Since we work with graded objects, we will pay a special attention to signs. Recall that if $P=\left\{P_{i}\right\}$ and $N=\left\{N_{i}\right\}$ are differential graded vector spaces with $\partial_{P}: P_{i} \rightarrow P_{i-1}$ and $\partial_{N}: N_{i} \rightarrow N_{i-1}$ then

- $P \otimes N$ is a differential graded vector space:

$$
(P \otimes N)_{r}=\bigoplus_{p+q=r} P_{p} \otimes N_{q}, \quad \partial_{P \otimes N}=\partial_{P} \otimes \operatorname{id}_{N}+\operatorname{id}_{P} \otimes \partial_{N},
$$

- $\operatorname{Hom}(P, N)$ is a differential graded vector space:

$$
\operatorname{Hom}_{n}(P, N)=\prod_{k-l=n} \operatorname{Hom}\left(P_{l}, N_{k}\right), \quad D_{\operatorname{Hom}(P, N)} f=\partial_{N} \circ f-(-1)^{|f|} f \circ \partial_{P} .
$$

The same formulae hold if $P=\left\{P^{i}\right\}$ and $N=\left\{N^{i}\right\}$ are differential graded vector spaces with $d_{P}: P^{i} \rightarrow P^{i+1}$ and $d_{N}: N^{i} \rightarrow N^{i+1}$. Later on we will omit subscripts on the differentials.

\subsection{Differential graded algebras, coalgebras and Lie algebras}

For precise definitions we refer to [12, $\$ 3 \mathrm{c}, \mathrm{d}$ and $\S 21]$. Recall that $T V$ denotes the tensor algebra on $V$, while $T^{c}(V)$ is the free supplemented coalgebra generated by $V$. If $C$ is a differential graded coalgebra with coproduct $\Phi$ and $A$ is a differential graded algebra with product $\mu$, then the cup product, $f \cup g=\mu \circ(f \otimes g) \circ \Phi$, gives the differential graded vector space $\operatorname{Hom}(C, A)$ a structure of differential graded algebra.

\subsection{Sullivan rational homotopy theory}

We refer the reader to [12, §12] for notation, terminology and results concerning Sullivan models. However, we recall here that if $V=\left\{V^{i}\right\}_{i \geq 0}$ is a graded $\mathbb{Q}$-vector space we denote by $\wedge V$ the free graded commutative algebra generated by $V$. Any path-connected space $X$ admits a Sullivan model

$$
\rho_{X}: \mathfrak{M}_{X}:=(\bigwedge V, d) \stackrel{\simeq}{\rightarrow} A_{P L}(X)
$$

where $A_{P L}$ denotes the contravariant functor of piecewise linear differential forms, and $\rho_{X}$ is a quasi-isomorphism [12, $\S 10$ and $\left.\S 12\right]$. If $X$ and $Y$ are two path-connected spaces then any continuous map $f: X \rightarrow Y$ admits a Sullivan representative $\mathfrak{M}_{f}$. Hereafter we will make the following identifications:

$$
H^{*}(X)=H\left(A_{P L}(X)\right)=H\left(\mathfrak{M}_{X}\right), \quad H^{*}(f)=H\left(A_{P L}(f)\right)=H\left(\mathfrak{M}_{f}\right) .
$$




\subsection{Semifree modules}

Let $A$ be a differential graded algebra. A differential graded $A$-module $P$ is called semifree if $P$ is equipped with a filtration $P=\bigcup_{n \geq 0} P(n)$ satisfying $P(0)=0, P(n) \subset$ $P(n+1)$ and such that $P(n) / P(n-1)$ is free on a basis of cycles [12, §6].

For any $A$-module $N$, there exists a semifree module $P$ and a quasi-isomorphism $\varphi: P \rightarrow N$. The module $P$ is called a semifree resolution of $N$.

\section{The dual of the loop product}

\subsection{A convenient definition of the dual of the loop product}

While M. Chas and D. Sullivan [6] have defined the loop product by using "transversal geometric chains" it is convenient for our purpose to define directly the dual of the loop product in the following way. First we replace the space $L M$ (respectively $M^{I}$ and $\Omega M$ ) by a Hilbert manifold ([1] or [7, Proposition 2.3.1]). Secondly, we consider the commutative diagram

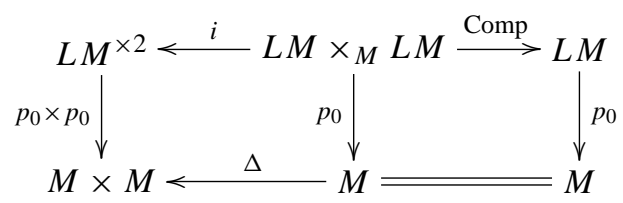

where

- Comp denotes composition of free loops,

- $p_{0}$ is the evaluation at 0 and is thus a locally trivial fibre bundle [3],

- the left hand square is a pullback diagram of locally trivial fibrations,

- the inclusion $i$ is the smooth embedding of the Hilbert manifold of composable loops into the product of the two Hilbert manifolds $L M \times L M$.

The embeddings $\Delta$ and $i$ both have codimension $m$. Thus, using the Thom-Pontryagin theory (see 4.5 for a precise definition), we obtain the Gysin maps

$$
\Delta^{!}: H^{k}(M) \rightarrow H^{k+m}\left(M^{\times 2}\right), \quad i^{!}: H^{k}\left(L M \times_{M} L M\right) \rightarrow H^{k+m}\left(L M^{\times 2}\right) .
$$

Thus diagram (1) yields the following diagram:

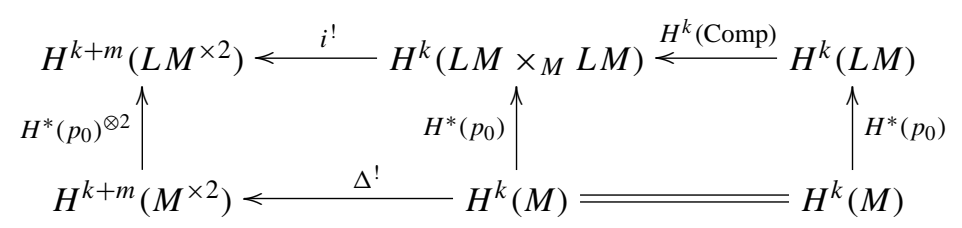

Following [22], [8] or [9], the dual of the loop product is defined by composition of maps in the upper line:

$$
i^{!} \circ H^{*}(\mathrm{Comp}): H^{*}(L M) \rightarrow H^{*+m}\left(L M^{\times 2}\right)
$$


while the map in the lower line is the dual of the intersection product. The commutativity of this diagram expresses the fact that $H^{*}\left(p_{0}\right)$ is a homomorphism between two cocommutative graded coalgebras.

\subsection{Why is it possible to express this definition in terms of Sullivan models?}

First we remark that diagram (1) is the pullback diagram of the diagram

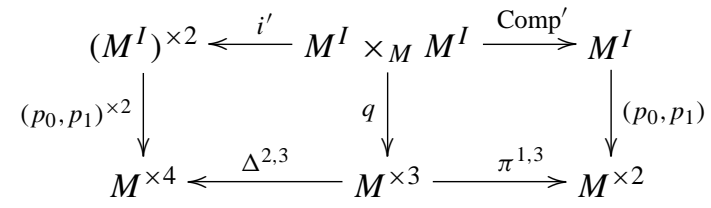

along the diagram

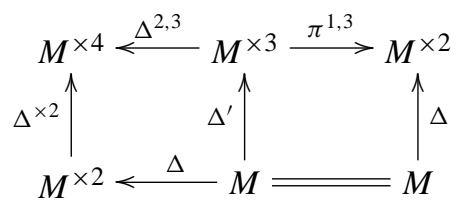

where $\Delta^{\prime}=(\Delta \otimes \mathrm{id}) \circ \Delta, \pi^{1,3}(x, y, z)=(x, z), q(\alpha, \beta)=(\alpha(0), \alpha(1)=\beta(0), \beta(1))$ and $\Delta^{2,3}(x, y, z)=(x, y, y, z)$. The other maps are the obvious ones. The second observation that once again the Thom-Pontryagin theory yields the commutative diagram

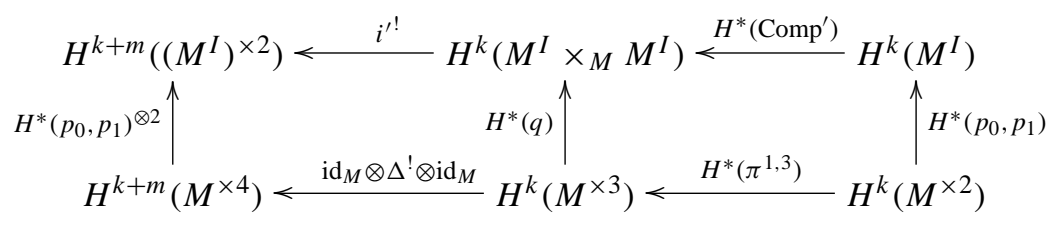

with $H^{*}\left(\pi^{1,3}\right)(a \otimes b)=a \otimes 1 \otimes b$.

It is then natural to define the path product as the composition of the maps in the upper line of the diagram $\left(2^{\prime}\right)$.

Since the loop product appears as a "pullback" of the path product it is worthwhile to determine the path product. This is the purpose of the last observation: There is a commutative diagram

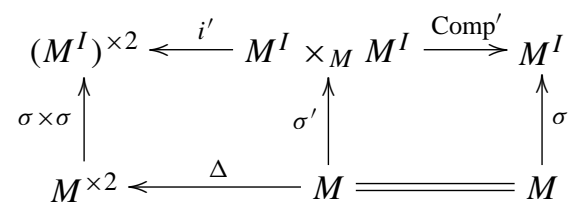


where $\sigma(x)$ is the constant path at $x \in M$ and $\sigma^{\prime}(x)=(\sigma(x), \sigma(x))$. From the properties of the Thom-Pontryagin construction, diagram (3) converts into a commutative diagram

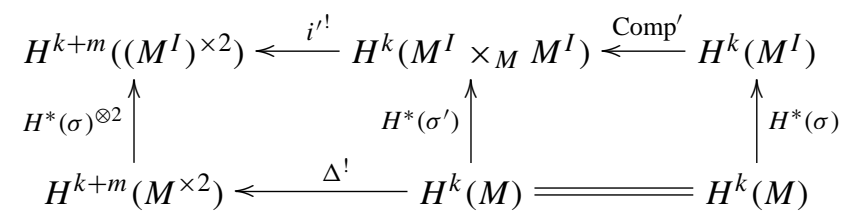

Since $\Delta^{!}$is the multiplication by the Euler class of the diagonal embedding (see 4.3) and since $\sigma$ is a homotopy equivalence we obtain a description of the path product in terms of Sullivan models.

\section{Theorem A. The dual of the loop product in terms of Sullivan models}

\subsection{Relative Sullivan model for loop fibrations}

It is convenient to consider a cofibrant Sullivan representative of a map $f: X \rightarrow Y$ called a relative Sullivan model [12, §14]: $\lambda_{f}: \mathfrak{M}_{Y} \hookrightarrow\left(\mathfrak{M}_{Y} \otimes \wedge V, d\right)=\mathfrak{M}_{X}$. Our primary example is the following diagram:

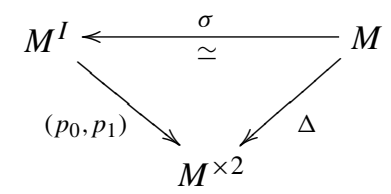

which describes the fibration associated to the diagonal map, and where $\sigma(x)$ is the constant path at $x \in M$. This diagram converts into

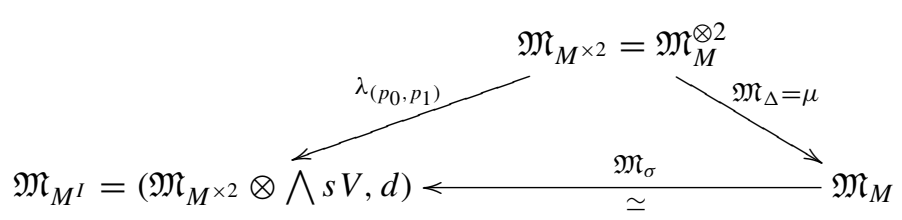

In this diagram:

a) $\mathfrak{M}_{M}=(\bigwedge V, d)$ is a Sullivan model of $M$.

b) $\mu$ is the product on $\bigwedge V$.

c) The differential in $\left.(\bigwedge V)^{\otimes 2} \otimes \bigwedge s V, d\right)=\mathfrak{M}_{M^{I}}$ is defined as in [12, §15, Example 1]: for $v \in V, s v \in s V$,

$$
\left\{\begin{array}{l}
d(v \otimes 1 \otimes \overline{1})=d v \otimes 1 \otimes \overline{1}, \\
d(1 \otimes v \otimes \overline{1})=1 \otimes d v \otimes \overline{1}, \\
d(1 \otimes 1 \otimes s v)=(v \otimes 1-1 \otimes v) \otimes \overline{1}-\sum_{i=1}^{\infty} \frac{(s d)^{i}}{i !}(v \otimes 1 \otimes \overline{1}) .
\end{array}\right.
$$


Here 1 and $\overline{1}$ denote respectively the unit of $\bigwedge V$ and of $\bigwedge s V$, and $s$ is the unique degree -1 derivation of $\bigwedge V \otimes \bigwedge V \otimes \bigwedge s V$ defined by

$$
\left\{\begin{array}{l}
s(v \otimes 1 \otimes \overline{1})=1 \otimes 1 \otimes s v=s(1 \otimes v \otimes \overline{1}), \\
s(1 \otimes 1 \otimes s v)=0 .
\end{array}\right.
$$

d) $\mathfrak{M}_{\sigma}=\mu \otimes \bar{\varepsilon}:\left((\bigwedge V)^{\otimes 2} \otimes \bigwedge s V, d\right)=\mathfrak{M}_{M^{I}} \rightarrow \mathfrak{M}_{M}=(\bigwedge V, d)$ is a quasiisomorphism with $\bar{\varepsilon}$ the canonical augmentation of $\bigwedge s V$.

The use of relative Sullivan models is interesting because it converts a pullback diagram of fibrations into a pushout diagram in the category of differential graded commutative algebras [12, Proposition 15.8]: Each pullback diagram

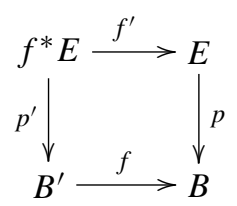

where $p$ is any fibration, converts into the pushout diagram in the category of differential graded commutative algebras,

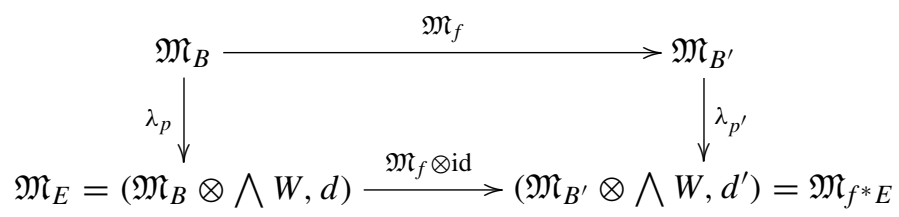

The differential on $\mathfrak{M}_{f^{*} E}$ is explicitly defined by the canonical isomorphism of graded algebras

$$
\mathfrak{M}_{B^{\prime}} \otimes \mathfrak{M}_{B} \mathfrak{M}_{E}=\mathfrak{M}_{B^{\prime}} \otimes \mathfrak{M}_{B}\left(\mathfrak{M}_{B} \otimes \wedge W\right) \stackrel{\cong}{\rightarrow} \mathfrak{M}_{B^{\prime}} \otimes \bigwedge W=\mathfrak{M}_{f^{*} E}
$$

As a first example, the pullback diagram

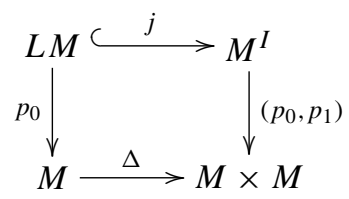

converts into the pushout diagram

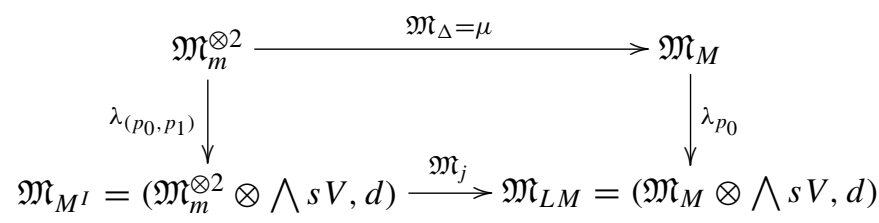


where $\lambda_{p_{0}}$ is a Sullivan relative model for $p_{0}[12, \S 12, \mathrm{c}]$. The differential on $\mathfrak{M}_{L M}=$ $(\bigwedge V \otimes \bigwedge s V, d)$ is defined by $d s=-s d$ where $s$ denotes the degree -1 derivation which extends the linear isomorphism $V \rightarrow s V$ and satisfies $s \circ s=0$. In particular $\mathfrak{M}_{j}=\mu \otimes \mathrm{id}$.

In the same way, the pullback diagram

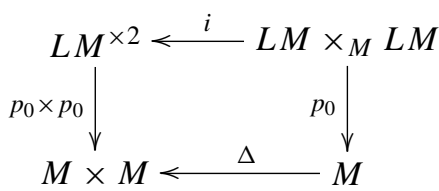

converts into the pushout diagram

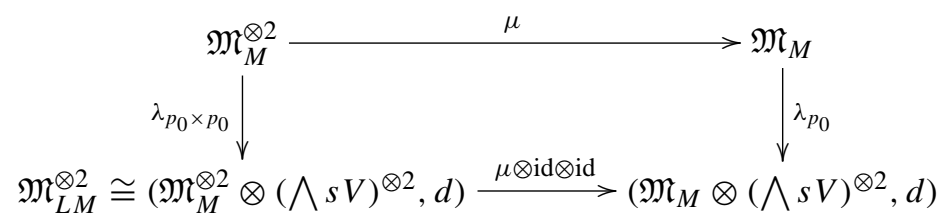

The differential on $\mathfrak{M}_{L M \times_{M} L M}=\left(\mathfrak{M}_{M} \otimes(\bigwedge s V)^{\otimes 2}, d\right)$ is defined by

$$
\left\{\begin{array}{l}
d(v \otimes \overline{1} \otimes \overline{1})=d v \otimes \overline{1} \otimes \overline{1}, \\
d(1 \otimes s v \otimes \overline{1})=-s(d v \otimes \overline{1} \otimes \overline{1}), \\
d(1 \otimes \overline{1} \otimes s v)=-s^{\prime}(d v \otimes \overline{1} \otimes \overline{1}),
\end{array}\right.
$$

Here $s$ and $s^{\prime}$ are the unique degree -1 derivations of $\bigwedge V \otimes(\bigwedge s V)^{\otimes 2}$ such that $s \circ s=$ $0=s^{\prime} \circ s^{\prime}$ and for $v \in V, s v \in s V$,

$$
\left\{\begin{array}{l}
s(v \otimes \overline{1} \otimes \overline{1})=1 \otimes s v \otimes \overline{1} \\
s^{\prime}(v \otimes \overline{1} \otimes \overline{1})=1 \otimes \overline{1} \otimes s v \\
s(1 \otimes s v \otimes \overline{1})=0=s^{\prime}(1 \otimes s v \otimes \overline{1}) \\
s(1 \otimes \overline{1} \otimes s v)=0=s^{\prime}(1 \otimes \overline{1} \otimes s v) .
\end{array}\right.
$$

These two examples provide us with relative Sullivan models for $L M \stackrel{p_{0}}{\rightarrow} M$ and $L M \times_{M} L M \stackrel{p_{0}}{\rightarrow} M$ as well as for $\mathfrak{M}_{i}=\mu \otimes \mathrm{id} \otimes \mathrm{id}: \mathfrak{M}_{L M} \rightarrow \mathfrak{M}_{L M \times_{M} L M}=$ $\mathfrak{M}_{L M} \otimes_{\mathfrak{M}_{M}} \mathfrak{M}_{L M}$

In a similar way, the pullback diagram which appears as the left part in diagram $\left(1^{\prime}\right)$ call it (7) - converts into the pushout diagram

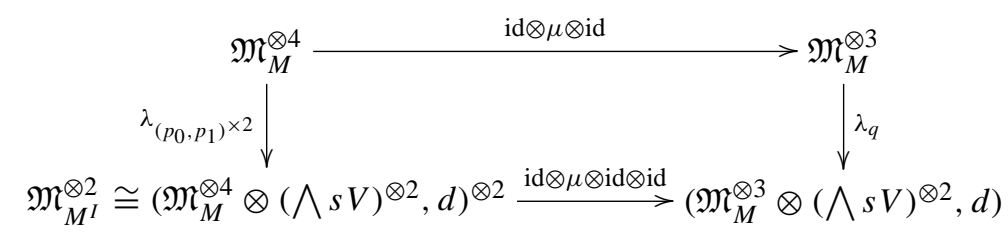


where the differential on $\mathfrak{M}_{M^{I} \times{ }_{M} M^{I}}=\left(\mathfrak{M}_{M}^{\otimes 3} \otimes(\bigwedge s V)^{\otimes 2}, d\right)$ is defined for $v \in V$ and $\bar{v} \in \bar{V}$ by:

$$
\left\{\begin{array}{l}
d(v \otimes 1 \otimes 1 \otimes \overline{1} \otimes \overline{1})=d v \otimes 1 \otimes 1 \otimes \overline{1} \otimes \overline{1} \\
d(1 \otimes v \otimes 1 \otimes \overline{1} \otimes \overline{1})=1 \otimes d v \otimes 1 \otimes \overline{1} \otimes \overline{1} \\
d(1 \otimes 1 \otimes v \otimes \overline{1} \otimes \overline{1})=1 \otimes 1 \otimes 1 \otimes d v \otimes \overline{1} \otimes \overline{1}, \\
d(1 \otimes 1 \otimes 1 \otimes s v \otimes \overline{1})=(v \otimes 1-1 \otimes v) \otimes 1 \otimes \overline{1} \otimes \overline{1}-\sum_{i=1}^{\infty} \frac{(s d)^{i}}{i !}(v \otimes 1 \otimes 1 \otimes \overline{1} \otimes \overline{1}), \\
d(1 \otimes 1 \otimes 1 \otimes \overline{1} \otimes s v)=1 \otimes(v \otimes 1-1 \otimes v) \otimes \overline{1} \otimes \overline{1}-\sum_{i=1}^{\infty} \frac{\left(s^{\prime} d\right)^{i}}{i !}(1 \otimes v \otimes 1 \otimes \overline{1} \otimes \overline{1}) .
\end{array}\right.
$$

Here $s$ and $s^{\prime}$ are the unique degree -1 derivations of $(\bigwedge V)^{\otimes 3} \otimes(\bigwedge s V)^{\otimes 2}$ such that $s \circ s=0=s^{\prime} \circ s^{\prime}$ and

$$
\left\{\begin{array}{l}
s(v \otimes 1 \otimes 1 \otimes \overline{1} \otimes \overline{1})=1 \otimes 1 \otimes 1 \otimes s v \otimes \overline{1}=s(1 \otimes v \otimes 1 \otimes \overline{1} \otimes \overline{1}), \\
s(1 \otimes 1 \otimes v \otimes \overline{1} \otimes \overline{1})=0=s^{\prime}(v \otimes 1 \otimes 1 \otimes \overline{1} \otimes \overline{1}), \\
s^{\prime}(1 \otimes v \otimes 1 \otimes \overline{1} \otimes \overline{1})=1 \otimes 1 \otimes 1 \otimes \overline{1} \otimes s v=s(1 \otimes 1 \otimes v \otimes \overline{1} \otimes \overline{1}) .
\end{array}\right.
$$

In particular $\mathfrak{M}_{i^{\prime}}=\mathrm{id} \otimes \mu \otimes$ id $\otimes$ id.

\subsection{Sullivan representatives of compositions of free paths and free loops}

Consider the next diagram which relates the right parts of diagrams $(1)$ and $\left(1^{\prime}\right)$ :

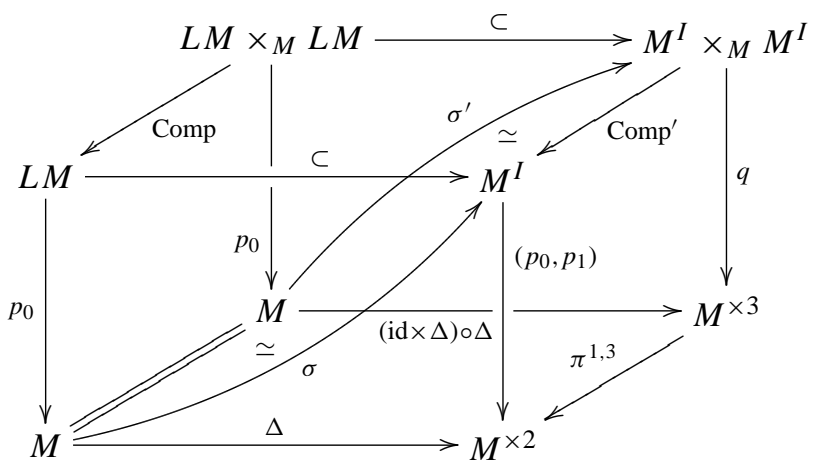

Lemma 1. There exists a unique homomorphism $\mathfrak{M}_{\mathrm{Comp}}$ of differential graded algebras such that, in the next diagram, the upper square commutes while the lower square com- 
mutes up to homotopy:

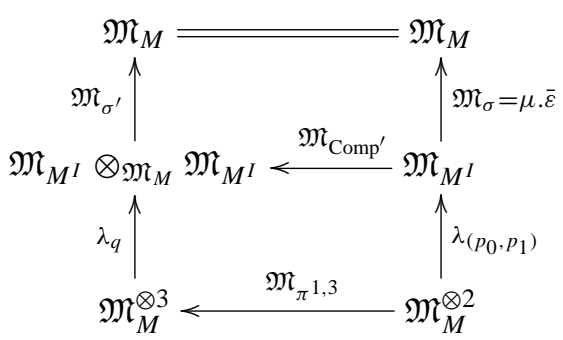

Moreover, $\mathfrak{M}_{\sigma^{\prime}}=\mu . \bar{\varepsilon} \otimes \mu . \bar{\varepsilon}$ is a surjective quasi-isomorphism.

Proof. Let us introduce the following notation:

a) $\bar{V}=s V, \hat{V}=V$,

b) $(\bigwedge \bar{V} \otimes \bigwedge \hat{V}, d)$ is the acyclic Sullivan algebra whose differential $d$ is defined by $d(\bar{v})=\hat{v}$ and $d(\hat{v})=0$

c) $\pi:(\bigwedge V \otimes \bigwedge \bar{V} \otimes \bigwedge \hat{V}, d) \rightarrow(\bigwedge V, d)$ is the quasi-isomorphism defined by $\pi(v)=$ $v$ and $\pi(\bar{v})=\pi(\hat{v})=0$,

d) $s$ is the derivation on $(\bigwedge V, d) \otimes(\bigwedge \bar{V} \otimes \bigwedge \hat{V}, d)$ defined by $s(v)=\bar{v}, s(\bar{v})=s(\hat{v})=0$.

Following [12, $\$ 15$ Example 1], there is an isomorphism $\varphi$ defined by

$$
\varphi: \mathfrak{M}_{M^{I}} \rightarrow(\bigwedge V, d) \otimes(\bigwedge \bar{V} \otimes \bigwedge \hat{V}, d),\left\{\begin{array}{l}
\varphi(v \otimes 1 \otimes \overline{1})=v, \\
\varphi(1 \otimes v \otimes \overline{1})=v+\hat{v}+\sum_{n=1}^{\infty} \frac{(s d)^{n}}{n !}(v), \\
\varphi(1 \otimes 1 \otimes s v)=\bar{v} .
\end{array}\right.
$$

This isomorphism gives the relative Sullivan model of $\left(p_{0}, p_{1}\right)$ considered in diagram (4). The composite

$$
\begin{aligned}
& \mathfrak{M}_{M^{I}} \otimes_{\mathfrak{M}_{M}} \mathfrak{M}_{M^{I}} \stackrel{\varphi \otimes_{\mathfrak{M}_{M}} \varphi}{\longrightarrow}(\bigwedge V, d) \otimes(\bigwedge \bar{V} \otimes \bigwedge \hat{V}, d) \otimes \wedge V(\bigwedge V, d) \otimes(\bigwedge \bar{V} \otimes \bigwedge \hat{V}, d) \\
& \downarrow i d \otimes \wedge V \pi \\
& (\bigwedge V, d) \otimes(\bigwedge \bar{V} \otimes \bigwedge \hat{V}, d) \otimes \wedge V(\bigwedge V, d) \\
& (\bigwedge V, d) \longleftarrow \text { id } \pi \cdot \pi
\end{aligned}
$$

is precisely $\mathfrak{M}_{\sigma^{\prime}}$. From the lifting lemma [12, Lemma 12.4], we deduce that there exists a unique homomorphism $\mathfrak{M}_{\text {Comp }}$ such that $\mathfrak{M}_{\text {Comp }} \circ \mathfrak{M}_{\sigma^{\prime}}=\mathfrak{M}_{\sigma}$. It follows from [12, Proposition 14.6] that the lower square of (9) is commutative up to homotopy. 
Therefore, using diagram $(\mathfrak{M}(5))$ of 4.1 , we obtain the commutative diagram

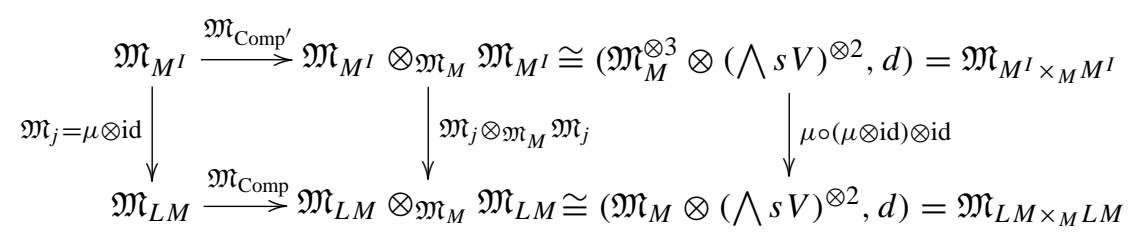

Thus we have proved:

Proposition 1. The homomorphism $\mathfrak{M}_{\text {Comp }}$ is a Sullivan representative of Comp : $L M \times{ }_{M} L M \rightarrow L M$.

\subsection{A representative for $i^{!}: H^{*}\left(L M \times_{M} L M\right) \rightarrow H^{*+m}(L M)^{\otimes 2}$}

First recall [2, VI, Theorem 12.4] that the Euler class of the diagonal embedding $\Delta$ : $M \rightarrow M \times M$ (also called the diagonal class) is the cohomology class

$$
e_{\Delta}=\sum_{l}(-1)^{\left|\beta_{l}\right|} \hat{\beta}_{l} \otimes \beta_{l} \in H^{m}(M \times M)=\left(H^{*}(M) \otimes H^{*}(M)\right)^{m}
$$

where $\left\{\beta_{l}\right\}$ denotes a homogeneous linear basis of $H^{*}(M)$ and $\left\{\hat{\beta}_{l}\right\}$ its Poincaré dual basis $\left(\left\langle\beta_{i} \cup \hat{\beta}_{j},[M]\right\rangle=\delta_{i}^{j} 1\right)$.

Observe here that $H^{*}(M)$ is an $H^{*}(M)^{\otimes 2}$-module via the multiplication $H^{*}(\Delta)$. Thus $\Delta^{!}$is mutiplication by $e_{\Delta}$ and an $H^{*}(M)^{\otimes 2}$-linear map.

The crucial point in our construction of a representative of $i !$ is the following result:

Proposition 2. There exists an $\mathfrak{M}_{M}^{\otimes 2}$-linear map

$$
f: \mathfrak{M}_{M^{I}}=\left(\mathfrak{M}_{M}^{\otimes 2} \otimes \bigwedge s V, d\right) \rightarrow \mathfrak{M}_{M}^{\otimes 2}
$$

of degree $m$ such that $f(d(x))=(-1)^{m} d \circ f(x)$. Moreover, $f$ is unique up to an $\mathfrak{M}_{M}^{\otimes 2}$ linear homotopy and

$$
\Delta^{!}=H(f) \circ H(\sigma)^{-1}
$$

Proof. The homomorphism defined in 4.1, $\mathfrak{M}_{\sigma}: \mathfrak{M}_{M^{I}}=\left(\mathfrak{M}_{M}^{\otimes 2} \otimes \wedge s V, d\right) \rightarrow \mathfrak{M}_{M}$, is an $\mathfrak{M}_{M}^{\otimes 2}$-semifree resolution (see 2.4). Thus (see [11, Appendix]) we have the Moore spectral sequence

$$
\operatorname{Ext}_{H \otimes H}^{p, q}(H, H \otimes H) \Rightarrow \operatorname{Ext}_{\mathfrak{M}_{M}^{\otimes 22}}^{p+q}\left(\mathfrak{M}_{M}, \mathfrak{M}_{M}^{\otimes 2}\right):=H^{*}\left(\operatorname{Hom}_{\mathfrak{M}_{M}^{\otimes 2}}\left(\mathfrak{M}_{M^{I}}, \mathfrak{M}_{M}\right)\right)
$$

with $H=H(\bigwedge V, d)$. The spectral sequence can also be constructed by replacing the minimal models by Halperin-Stasheff filtered models ([16]) and by using the induced filtration on the Hom complex. Since $H \otimes H$ is a Poincaré duality algebra of formal 
dimension $2 m$, by [11, Theorem 3.1] we have

$$
\operatorname{Ext}_{H \otimes H}^{p, q}(\mathbb{Q}, H \otimes H) \cong \begin{cases}\{0\} & \text { if } p+q \neq 2 m \\ \mathbb{Q} & \text { if } p+q=2 m\end{cases}
$$

Induction on the dimension of $E$ proves that $\operatorname{Ext}_{H \otimes H}^{p, q}(E, H \otimes H)=0$ if $0 \leq p+q<$ $2 m-d$ for any finite-dimensional $H \otimes H$-module $E$ concentrated in degrees $\leq d$. In particular,

$$
\operatorname{Ext}_{H \otimes H}^{p, q}(H, H \otimes H)=0 \quad \text { if } 0 \leq p+q<m .
$$

On the other hand, since $M$ is simply connected, we have $H^{m-1}=0$, and from the long exact sequence associated to the short exact sequence $0 \rightarrow H^{m} \rightarrow H \rightarrow H / H^{m} \rightarrow 0$ we deduce that

$$
\operatorname{Ext}_{H \otimes H}^{p, q}(H, H \otimes H) \cong \begin{cases}\{0\} & \text { if } p+q=m+1, \\ \mathbb{Q} & \text { for }(p, q) \text { such that } p+q=m .\end{cases}
$$

Conditions $\left(C_{1}\right)$ and $\left(C_{2}\right)$ and the convergence of the Moore spectral sequence imply that

$$
\operatorname{Ext}_{\mathfrak{M}_{M}^{\otimes 2}}^{m}\left(\mathfrak{M}_{M}, \mathfrak{M}_{M}^{\otimes 2}\right) \cong \mathbb{Q}
$$

Multiplication by $e_{\triangle}$ defines a generator of $\operatorname{Ext}_{H \otimes H}^{m}(H, H \otimes H)$ which survives in $\operatorname{Ext}_{\mathfrak{M}_{M}^{\otimes 2}}^{m}\left(\mathfrak{M}_{M}, \mathfrak{M}_{M}^{\otimes 2}\right) \cong \mathbb{Q}$. Any cocycle in $\operatorname{Hom}_{\mathfrak{M}_{M}^{\otimes 2}}\left(\mathfrak{M}_{M^{I}}, \mathfrak{M}_{M}\right)$ can be viewed as a map $f$ satisfying the conclusion of Proposition 2. If $f$ and $f^{\prime}$ are two such cocycles then $f-f^{\prime}=D h$ and $h$ is the required homotopy between $f$ and $f^{\prime}$.

The proof of the next result is postponed to Subsection 4.5.

Proposition 3. The map $\mathfrak{M}_{i}^{!}$, defined as the composition of the following natural maps:

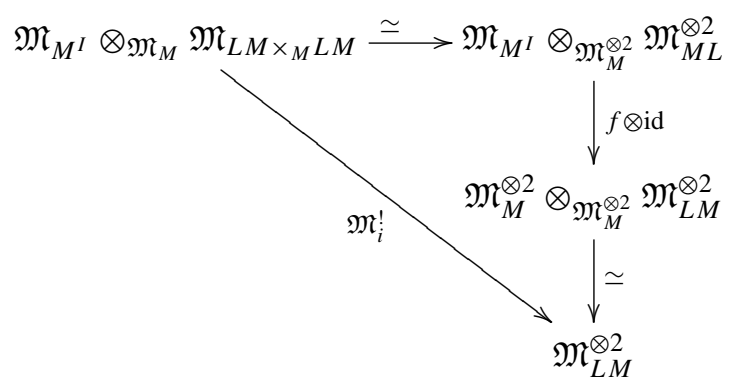

is such that

$$
i^{!}=H\left(\mathfrak{M}_{i}^{!}\right) \circ H\left(\mathfrak{M}_{\sigma} \otimes \mathrm{id}\right)^{-1}: H^{k}\left(L M \times{ }_{M} L M\right) \rightarrow H^{*+m}\left(L M^{\times 2}\right) .
$$




\subsection{Statement of Theorem A and Examples}

From diagram (2) and Propositions 1 and 3 we deduce our first result:

Theorem A. The dual of the loop product

$$
H^{*}(L M) \stackrel{H^{*}(\mathrm{Comp})}{\longrightarrow} H^{*}\left(L M \times_{M} L M\right) \stackrel{i^{!}}{\rightarrow} H^{*+m}\left(L M^{\times 2}\right)
$$

is induced in homology by the maps

$$
\mathfrak{M}_{L M \times_{M} L M}=\mathfrak{M}_{M} \otimes_{\mathfrak{M}_{M}} \mathfrak{M}_{L M \times{ }_{M} L M} \stackrel{\mathfrak{M}_{L M}}{\stackrel{\mathfrak{M}_{\sigma} \otimes \mathrm{id}}{\simeq}} \mathfrak{M}_{M^{I}} \otimes_{\mathfrak{M}_{M}} \mathfrak{M}_{L M \times_{M} L M} \simeq \mathfrak{M}_{L M \times_{M} L M}
$$

Example: Formal spaces. Let $M$ be a formal space, i.e. a space $M$ whose minimal model $\mathfrak{M}_{M}=(\bigwedge V, d)$ is quasi-isomorphic to $\left(H=H^{*}(M), 0\right)$. Examples of formal spaces are given by connected compact Kähler manifolds ([10]) and quotients of compact connected Lie groups by closed subgroups of the same rank. When $M$ is a formal space the dual of the loop product is induced in homology by the maps

$$
\begin{gathered}
(H \otimes \wedge s V, D) \\
H\left(\mathfrak{M}_{\text {Comp }}\right) \downarrow \\
\left(H \otimes(\bigwedge s V)^{\otimes 2}, d\right) \stackrel{\mu \cdot \bar{\varepsilon} \otimes \mathrm{id}}{\leftarrow}\left(H^{\otimes 2} \otimes \bigwedge s V\right) \otimes_{H}\left(H \otimes(\bigwedge s V)^{\otimes 2}\right) \\
\mathfrak{M}_{i}^{!} \downarrow \\
\left(H^{\otimes 2} \otimes(\bigwedge s V)^{\otimes 2}, d\right)
\end{gathered}
$$

Let us describe explicitly the particular case $M=\mathbb{C} P^{n}$. The minimal model of $\mathbb{C} P^{n}$ is given by $(\bigwedge(x, y), d), d(y)=x^{n+1},|x|=2,|y|=2 n+1$. Thus the relative Sullivan model of the free loop space is $(\bigwedge(x, \bar{x}, y, \bar{y}), d), d(\bar{x})=0, d(\bar{y})=-(n+1) x^{n} \bar{x}$. Since we have a quasi-isomorphism $(\bigwedge(x, y), d) \rightarrow\left(\bigwedge(x) /\left(x^{n+1}\right), d\right)=: H$, the space $\mathbb{C} P^{n}$ is formal. A linear basis of

$$
H^{*}(L M)=H\left(\bigwedge(x, \bar{x}, \bar{y}) /\left(y^{n+1}\right), d\right) \cong \mathbb{Q} \cdot 1 \oplus\left(\bigwedge^{\geq 1}(x, \bar{x}) /\left(x^{n+1}, x^{n} \bar{x}\right) \otimes \bigwedge \bar{y}\right)
$$

is formed by the elements $1, x^{p} \bar{y}^{[q]}$, and $x^{r} \bar{x} \bar{y}^{[s]}, p=1, \ldots, n, q \geq 0, s \geq 0, r=$ $0, \ldots, n-1$, with $\bar{y}^{[s]}=\bar{y}^{s} / s$ !. 
A Sullivan representative of the composition of free loops Comp : $L M \times_{M} L M$ $\rightarrow L M$ is given by

$$
\mathfrak{M}_{\text {Comp }}(\bar{x})=\bar{x}+\bar{x}^{\prime}, \quad \mathfrak{M}_{\text {Comp }}(\bar{y})=\bar{y}+\bar{y}^{\prime}-\frac{n(n+1)}{2} x^{n-1} \bar{x} \bar{x}^{\prime} .
$$

The dual of the loop product is induced by the map

$$
\begin{gathered}
\theta:\left(H^{*}(M) \otimes \wedge(\bar{x}, \bar{y}), d\right) \rightarrow\left(H^{*}(M) \otimes \wedge(\bar{x}, \bar{y}), d\right) \otimes\left(H^{*}(M) \otimes \wedge(\bar{x}, \bar{y}), d\right), \\
\left\{\begin{array}{c}
\theta\left(\alpha \otimes \bar{y}^{[s]}\right)=\sum_{p=0}^{n} \sum_{j=0}^{s} \alpha x^{p} \bar{y}^{[j]} \otimes x^{n-p} \bar{y}^{[s-j]} \\
-\frac{n(n+1)}{2} \sum_{p=0}^{n} \sum_{j=0}^{s-1} \alpha x^{n-1+p} \bar{x} \bar{y}^{[j]} \otimes x^{n-p} \bar{x} \bar{y}^{[s-j]}, \\
\theta\left(\alpha \otimes \bar{x} \otimes \bar{y}^{[s]}\right)=(1 \otimes \bar{x}+\bar{x} \otimes 1) \cdot\left(\sum_{p=0}^{n} \sum_{j=0}^{s} \alpha x^{p} \bar{y}^{[j]} \otimes x^{n-p} y^{[s-j]}\right),
\end{array}\right.
\end{gathered}
$$

with $\alpha \in H^{*}(M)$. The dual basis

$$
1, a_{p, q}, b_{r, s}, \quad p=1, \ldots, n, q \geq 0, s \geq 0, r=0, \ldots, n-1,
$$

with $\left|a_{p, q}\right|=2 p+2 q n,\left|b_{r, s}\right|=2 r+1+2 s n$, is a linear basis of $H_{*}(L M)$. Thus the loop product $H_{*}(L M \times L M) \rightarrow H_{*-m}(L M), x \otimes y \mapsto x \bullet y$, is described by the formulae

$a_{p, q} \bullet a_{r, s}=a_{p+r-n, q+s}, \quad a_{p, q} \bullet b_{r, s}=b_{p+r-n, q+s}, \quad\left(a_{n-1,0}\right)^{n}=1, \quad 1 \bullet a_{n, 1}=0$.

This shows that

$$
\mathbb{H}_{*}\left(L\left(\mathbb{C} P^{n}\right) ; \mathbb{Q}\right) \cong \bigwedge(a, b, t) /\left(a^{n+1}, a^{n} b, a^{n} t\right),
$$

with $|a|=-2,|b|=-1$ and $|t|=2 n, a=a_{n-1,0}, b=b_{n-1,0}, t=a_{n, 1}$ (cf. [9]).

\subsection{Proof of Proposition 3}

Let $M$ and $N$ be (smooth Banach and without boundary) connected manifolds and $f$ : $M \rightarrow N$ be a (smooth) closed embedding [18, II, §2]. Then we have the exact sequence of fiber bundles

$$
0 \rightarrow T M \stackrel{T f}{\rightarrow} T N_{\mid M} \rightarrow v_{f} \rightarrow 0
$$

where $T M$ and $T N$ are the tangent bundles and $v_{f}$ is the normal fiber bundle of $f$. By definition of an immersion, this exact sequence splits [18, II, Proposition 2.3]. Hereafter we will identify $v_{f}$ with a factor bundle of $T N_{\mid M}$. When the fiber of $v_{f}$ is of finite dimension $k$, the embedding has codimension $k$. Consider the associated disk and sphere bundles, $v^{D}, v^{S}$, and the Thom class of the oriented normal fiber bundle pair $\left(v^{D}, v^{S}\right)$. The exponential map $D \subset T N \rightarrow N$ restricted to $v_{f}$ is a local isomorphism on the zero 
section of the bundle $T N \rightarrow N$. Since $f$ is a closed embedding and since $N$ admits a partition of unity, by [18, IV, Theorem 5.1], there exists an open neighborhood $Z$ of the zero section of $v_{f}$, an open neighborhood $U$ of $f(M)$ in $N$, and an isomorphism $\theta: Z \rightarrow U$ which identifies the zero section of $v_{f}$ with $f(M)$. Since $f$ has finite codimension $k$ we identify $Z$ with $v_{f}^{D}$, and the isomorphism $v_{f}^{D} \stackrel{\theta}{\rightarrow} U=$ : tube $f$ restricts to an isomorphism $v_{f}^{S} \cong \theta\left(v_{f}^{S}\right)=$ : $\partial$ tube $f$. The above discussion is summarized in the commutative diagram

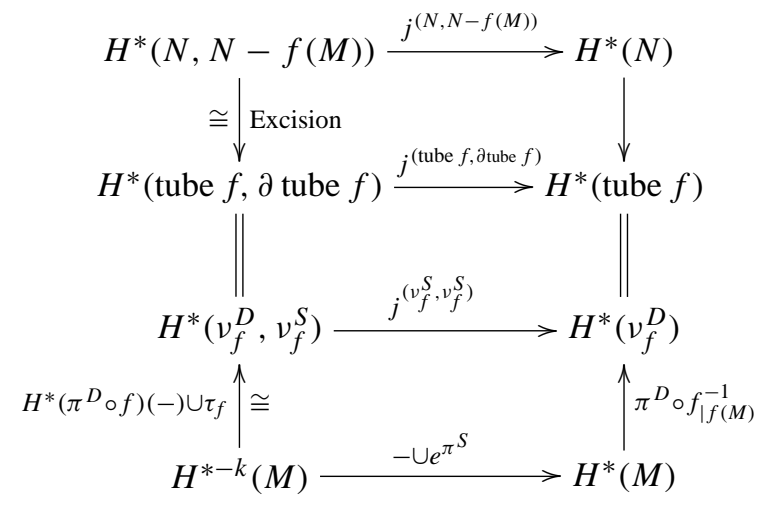

The $H^{*}(N)$-linear map $f^{!}$defined as the composition of the natural homomorphisms

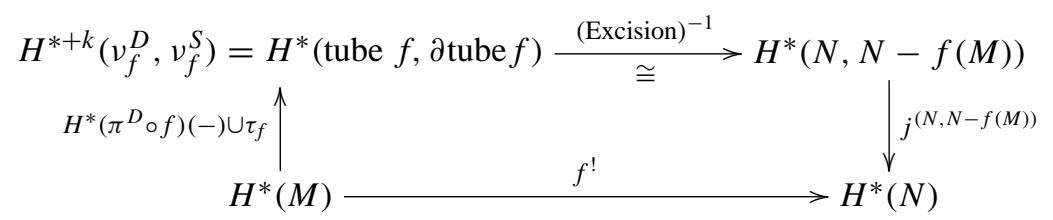

is called the cohomology Gysin map and $f^{!}(1)=e_{f} \in H^{k}(N)$ is called the Euler class of the embedding $f([20])$.

End of proof of Proposition 3. First consider the commutative diagram

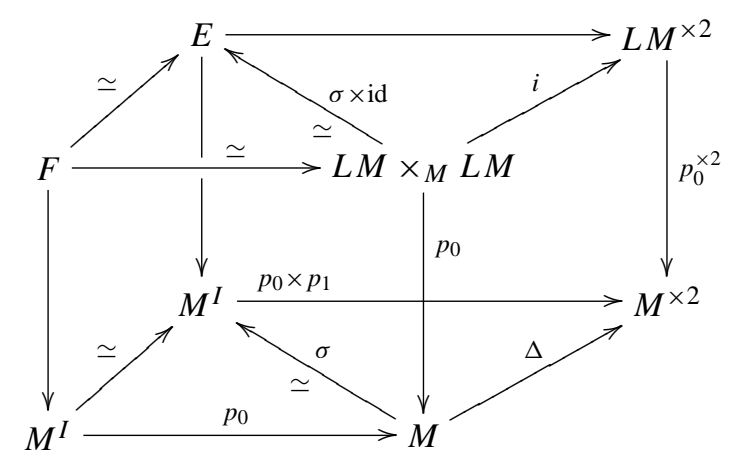

where the front face and the back face of the cube are pullback diagrams. 
Secondly, observe that

$$
H^{*}(E)=H\left(\mathfrak{M}_{M^{I}} \otimes_{\mathfrak{M}_{M}^{\otimes 2}} \mathfrak{M}_{L M}^{\otimes 2}\right) \quad \text { and } \quad H^{*}(F)=H\left(\mathfrak{M}_{M^{I}} \otimes_{\mathfrak{M}_{M}} \mathfrak{M}_{L M \times_{\mathfrak{M}_{M}} L M}\right) .
$$

From Proposition 2 we have the associated commutative diagram

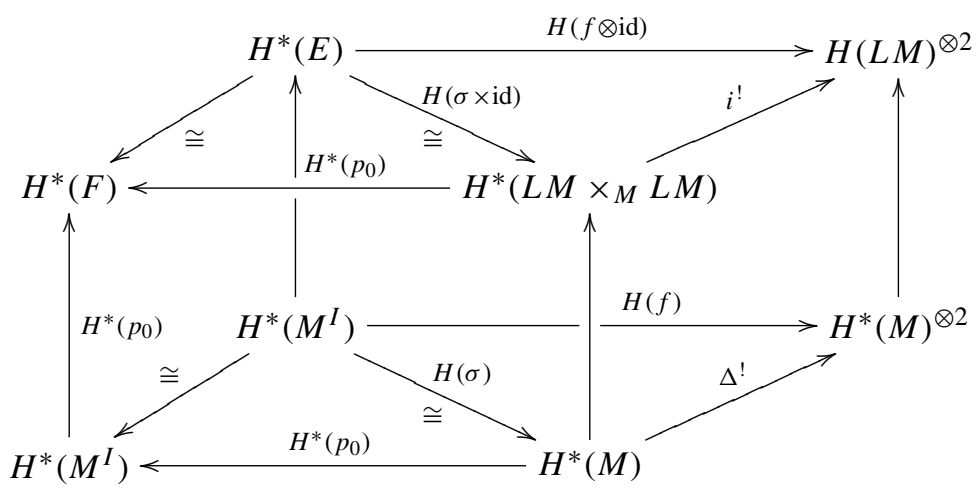

This ends the proof of Proposition 3.

\section{Theorem B. String bracket in terms of Sullivan models}

\subsection{Statement and proof of Theorem $B$}

The string homology (respectively string cohomology) is the desuspended equivariant homology of the free loop space (respectively the graded dual of the desuspended equivariant homology)

$$
\mathcal{H}_{*}=H_{*+m}^{S^{1}}(L M)=H_{*+m}\left(L M \times_{S^{1}} E S^{1}\right) \quad\left(\text { respectively } \mathcal{H}^{*}=\left(\mathcal{H}_{*}\right)^{\vee}\right) .
$$

Let $\xi \in H^{2}\left(L M \times{ }_{S^{1}} E S^{1}\right)$ be the characteristic class of the circle bundle $S^{1} \rightarrow$ $L M \times E S^{1} \stackrel{p}{\rightarrow} L M \times_{S^{1}} E S^{1}$ and consider the associated Gysin sequence

$$
\cdots \rightarrow \mathbb{H}_{n} \stackrel{H(p)}{\longrightarrow} \mathcal{H}_{n} \stackrel{\xi \cap-}{\longrightarrow} \mathcal{H}_{n-2} \stackrel{\mathbb{M}}{\longrightarrow} \mathbb{H}_{n-1} \rightarrow \cdots
$$

The string bracket on $\mathcal{H}_{*}$ is the bilinear map (see [6])

$$
[-,-]: \mathcal{H}_{*} \otimes \mathcal{H}_{*} \rightarrow \mathcal{H}_{*-2}, \quad a \otimes b \mapsto[a, b]=(-1)^{|a|} H_{*}(p)(\mathbb{M}(a) \bullet \mathbb{M}(b)),
$$

where $\bullet$ denotes the loop product on $\mathbb{H}_{*}(L M)$.

Let $\mathfrak{M}_{M} \stackrel{\lambda_{p_{0}}}{\hookrightarrow} \mathfrak{M}_{L M}=\left(\mathfrak{M}_{M} \otimes \wedge s V, d\right)$ be a Sullivan model of $L M \stackrel{p_{0}}{\rightarrow} M$ (see diagram (5)). Then a Sullivan representative for the inclusion $L M \rightarrow L M \times{ }_{S^{1}} E S^{1}$ is given by the $\mathfrak{M}_{L M}$-linear map (see [4])

$$
\pi:\left(\mathfrak{M}_{L M} \otimes \bigwedge u, D\right) \rightarrow \mathfrak{M}_{L M}, \quad 1 \otimes u \mapsto 0,
$$


where $\left(\mathfrak{M}_{L M} \otimes \bigwedge u, D\right)$ is a Sullivan model for the equivariant free loop space $L M \times_{S^{1}}$ $E S^{1}$ whose differential $D$ is given by $D(u)=0,|u|=2, D(v)=d(v)+u s(v), D(s v)=$ $-s(d v)$. This proves that the dual of the Gysin sequence (10) is the homology long exact sequence associated to the short exact sequence of cochain complexes

$$
0 \rightarrow\left(\mathfrak{M}_{L M} \otimes \bigwedge u, D\right) \stackrel{\mu_{u}}{\rightarrow}\left(\mathfrak{M}_{L M} \otimes \bigwedge u, D\right) \stackrel{\pi}{\rightarrow} \mathfrak{M}_{L M} \rightarrow 0 .
$$

The connecting map $\tilde{s}$ is induced by the derivation $s$ considered in the definition of $\mathfrak{M}_{L M}$ (see 4.1) and $\mu_{u}$ denotes multiplication by the cocycle $u$. Then Theorem B follows from Theorem A and the definition of the string bracket:

Theorem B. The dual of the string bracket

$$
\mathcal{B}^{\vee}: \mathcal{H}^{*} \rightarrow\left(\mathcal{H}^{*} \otimes \mathcal{H}^{*}\right)^{*+2}
$$

is induced in homology by the homomorphisms of complexes:

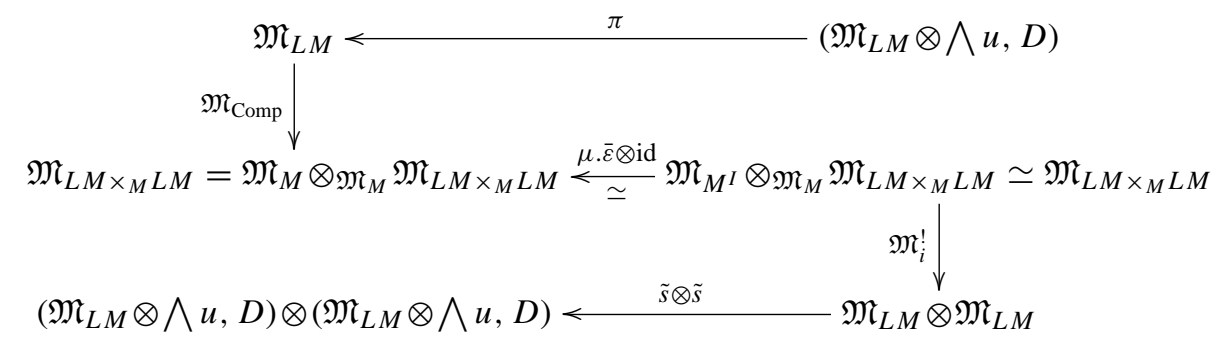

\subsection{Examples}

1) Assume that $H^{*}(M)$ is equal to $\bigwedge u$ with $|u|$ odd, or to $\bigwedge u /\left(u^{n+1}\right)$ with $|u|$ even. From [4], we have the following facts:

If $H^{*}(M)=\bigwedge u$ with $|u|=2 p+1$ then $\mathcal{H}_{2 i}=0$ for all $i$.

If $H^{*}(M)=\bigwedge u /\left(u^{n+1}\right)$ with $|u|=2 p$, then $\operatorname{dim}_{\mathbb{Q}} \mathcal{H}_{2 i}=1$ for all $i$.

Furthermore, the space $M$ is formal and it is shown in [23] that the map $\xi \cap-$ is an isomorphism.

This proves, in the two cases, the nullity of the maps

$$
\mathbb{E}=H_{*}(p): \mathbb{H}_{2 i} \rightarrow \mathcal{H}_{2 i} \quad \text { and } \quad \mathbb{M}: \mathcal{H}_{2 i} \longrightarrow \mathbb{H}_{2 i+1} \text {. }
$$

Let $a \in \mathcal{H}_{2 i-1}$ and $b \in \mathcal{H}_{2 j-1}$, for some $(i, j) \in \mathbb{Z}^{2}$. Then $\mathbb{M}(a) \bullet \mathbb{M}(b) \in \mathbb{H}_{2(i+j+1)}$, so we have $[a, b]=-\mathbb{E}(\mathbb{M}(a) \bullet \mathbb{M}(b))=0$. Thus, for such manifolds $M$ the string bracket is trivial.

2) If $M:=S^{2 k+1} \times S^{2 k+1}, k \geq 1$, then models for $M, L M$ and $L M \times_{S^{1}} E S^{1}$ are given by

$$
\begin{array}{ll}
M: & (\bigwedge(x, y), 0), \\
L M: & (\bigwedge(x, y, \bar{x}, \bar{y}), 0), \\
L M \times_{S^{1}} E S^{1}: & (\bigwedge(x, y, \bar{x}, \bar{y}, u), D),\left\{\begin{array}{l}
D u=D(\bar{x})=D(\bar{y})=0, \\
D y=u \bar{y}, \quad D x=u \bar{x} .
\end{array}\right.
\end{array}
$$


A set of cocycles representing a basis of the vector space $\tilde{H}_{S^{1}}^{*}(L M):=H_{S^{1}}^{*}(L M) / \mathbb{Q} u$ is formed by the elements $([4])$

$$
e_{a, b}=\bar{x}^{a} \bar{y}^{b}, \quad(a, b) \in \mathbb{N}^{2}-(0,0), \quad f_{a, b}=(y \bar{x}-x \bar{y}) \bar{x}^{a} \bar{y}^{b}, \quad(a, b) \in \mathbb{N}^{2} .
$$

The vector space $\tilde{H}^{*}(L M)$ has the following basis:

$$
\left\{\begin{array} { l } 
{ e _ { a , b } , } \\
{ f _ { a , b } , }
\end{array} \quad \left\{\begin{array}{ll}
e_{a, b}^{\prime}=x y \bar{x}^{a} \bar{y}^{b}, & (a, b) \in \mathbb{N}^{2}, \\
f_{a, b}^{\prime}=x \bar{x}^{a} \bar{y}^{b}, & (a, b) \in \mathbb{N}^{2}, \\
f_{b}^{\prime \prime}=y \bar{y}^{b}, & b \in \mathbb{N} .
\end{array}\right.\right.
$$

From the above description of the Gysin sequence we deduce $H^{*}(p)\left(e_{a, b}\right)=e_{a, b}$, $H^{*}(p)\left(f_{a, b}\right)=f_{a, b}, M^{\prime}\left(f_{a, b}^{\prime}\right)=e_{a+1, b}, M^{\prime}\left(e_{a, b}^{\prime}\right)=f_{a, b}, M^{\prime}\left(f_{b}^{\prime \prime}\right)=e_{0, b+1}, M^{\prime}\left(e_{a, b}\right)$ $=0$, and $M^{\prime}\left(f_{a, b}\right)=0$.

To fix signs, denote by $x y$ the fundamental class of $M$. A straightforward computation shows that

$$
\begin{aligned}
\mathcal{B}^{\vee}\left(u^{r}\right) & =0, \\
\mathcal{B}^{\vee}\left(\bar{x}^{p} \bar{y}^{q}\right) & =\sum_{r=0}^{p} \sum_{s=0}^{q}\left(\begin{array}{l}
p \\
r
\end{array}\right)\left(\begin{array}{l}
q \\
s
\end{array}\right)\left(\bar{x}^{r} \bar{y}^{s+1} \otimes \bar{x}^{p-r+1} \bar{y}^{q-s}-\bar{x}^{r+1} \bar{y}^{s} \otimes \bar{x}^{p-r} \bar{y}^{q-s+1}\right) \\
\mathcal{B}^{\vee}\left(f_{p, q}\right) & =\left(f_{0,0} \otimes 1+1 \otimes f_{0,0}\right)(\bar{x} \otimes \bar{y}-\bar{y} \otimes \bar{x}) \cdot \mathcal{B}^{\vee}\left(\bar{x}^{p} \bar{y}^{q}\right) .
\end{aligned}
$$

To describe the string bracket in $\mathcal{H}_{*}$ we choose the dual basis $t_{r}, a_{p, q}$ and $b_{p, q}$ to $u^{r}$, $\bar{x}^{p} \bar{y}^{q}$ and $f_{p, q}$. In that basis the string bracket satisfies

$$
\begin{aligned}
& {\left[b_{k, t}, a_{l, m}\right]=\left(\begin{array}{c}
k+l \\
k
\end{array}\right)\left(\begin{array}{c}
m+t \\
t
\end{array}\right) \frac{k m-l t}{(k+l)(t+m)} b_{k+l-1, t+m-1},} \\
& {\left[a_{k, t}, a_{l, m}\right]=\left(\begin{array}{c}
k+l \\
k
\end{array}\right)\left(\begin{array}{c}
m+t \\
t
\end{array}\right) \frac{l t-k m}{(k+l)(t+m)} a_{k+l-1, t+m-1},} \\
& {\left[b_{r, s}, b_{m, n}\right]=0 .}
\end{aligned}
$$

In particular the string Lie algebra $\mathcal{H}_{*}$ is not nilpotent, since for instance $\left[a_{1,1}, a_{r, s}\right]=$ $(r-s) a_{r, s}$.

\section{Theorem C. Dual of the loop product in terms of cochains on a differential graded Lie algebra}

\subsection{Chains and cochains on a differential graded Lie algebra}

Let $(L, \partial)$ be a differential graded Lie algebra with $\partial: L_{i} \rightarrow L_{i-1}$ and $(P, \partial)$ (respectively $(Q, \partial))$ be a left (respectively right) differential $L$-module. The two-sided chain complex $\mathcal{C}_{*}(P ; L ; Q)$ is defined as follows:

$$
\mathcal{C}_{k}(P ; L ; Q)=\left(P \otimes \bigwedge^{k} s L \otimes Q, \partial_{0}+\partial_{1}\right) .
$$


A generic element is written $p \otimes s x_{1} \wedge \cdots \wedge s x_{k} \otimes q$ with degree $|p|+|q|+\sum_{i=1}^{k}\left|s x_{i}\right|$. The differential $\partial=\partial_{0}+\partial_{1}$ is defined by

$$
\begin{gathered}
\mathcal{C}_{k}(P ; L ; Q) \stackrel{\partial_{0}}{\rightarrow} \mathcal{C}_{k}(P ; L ; Q), \quad \mathcal{C}_{k}(P ; L ; Q) \stackrel{\partial_{1}}{\rightarrow} \mathcal{C}_{k-1}(P ; L ; Q), \\
\partial_{0}(p \otimes c \otimes q)=\partial p \otimes s x_{1} \wedge \cdots \wedge s x_{k} \otimes q \\
\quad-p \otimes \sum_{i=1}^{k}(-1)^{|p|+\epsilon_{i}} s x_{1} \wedge \cdots \wedge s \partial x_{i} \wedge \cdots \wedge s x_{k} \otimes q \\
\quad+(-1)^{|p|+\epsilon_{k}} p \otimes c \otimes \partial q, \\
\partial_{1}\left(p \otimes s x_{1} \wedge \cdots \wedge s x_{k} \otimes q\right) \\
=\sum_{i=1}^{k}(-1)^{|p|+\left|s x_{i}\right| \epsilon_{i}} p \cdot x_{i} \otimes s x_{1} \wedge \cdots \widehat{s x_{i}} \cdots \wedge s x_{k} \otimes q \\
\quad+p \otimes \sum_{1 \leq i<j \leq k}(-1)^{|p|+\epsilon_{i j}} s\left[x_{i}, x_{j}\right] \wedge \cdots \widehat{s x_{i}} \cdots \widehat{s x_{j}} \cdots \wedge s x_{k} \otimes \partial q \\
\quad+\sum_{i=1}^{k}(-1)^{|p|+\epsilon_{k+1}+\left|x_{i}\right|+\left|s x_{i}\right| \epsilon_{k, i} p \otimes s x_{1} \wedge \cdots \widehat{s x_{i}} \cdots \wedge s x_{k} \otimes x_{i} \cdot q}
\end{gathered}
$$

where $\epsilon_{i, j}=\epsilon_{i+1}+\epsilon_{j}$ for $i<j$ and $\epsilon_{i}=\sum_{k<i}\left|s x_{k}\right|$.

The chain coalgebra of $(L, \partial)$ is the graded differential coalgebra $\mathcal{C}_{*}(L)=$ $\left(\bigwedge s L, \partial_{0}+\partial_{1}\right):=\mathcal{C}_{*}(\mathbb{Q} ; L ; \mathbb{Q})$ with coproduct

$$
s x_{1} \wedge \cdots \wedge s x_{k} \mapsto \sum_{j=0}^{k} \sum_{\sigma \in \operatorname{Sh}(j)} \varepsilon_{\sigma}\left(s x_{\sigma(1)} \wedge \cdots \wedge s x_{\sigma(j)}\right) \otimes\left(s x_{\sigma(j+1)} \wedge \cdots \wedge s x_{\sigma(k)}\right),
$$

where $\varepsilon_{\sigma}$ is the graded signature and $\operatorname{Sh}(j)$ denotes the set of $(j, k-j)$-shuffles.

Recall that if $Q$ is a left $L$-module then it is also a right $L$-module for the action defined by $a \cdot x:=-(-1)^{|a| \cdot|x|} x \cdot a, a \in Q, x \in U L$. For any left $L$-differential module $Q$ (respectively for any right differential module $P$ ) put

$$
\mathcal{C}_{*}(L ; Q)=\mathcal{C}_{*}(\mathbb{Q} ; L ; Q) \quad\left(\text { respectively } \mathcal{C}_{*}(P ; L)=\mathcal{C}_{*}(P ; L ; \mathbb{Q})\right) .
$$

Thus, as graded vector spaces, $\mathcal{C}_{*}(L ; Q)=\bigwedge s L \otimes Q$ and $\mathcal{C}_{*}(P ; L)=P \otimes \wedge s L$. The cochain complex of $L$ with coefficients in a right $L$-module $P$ is defined by

$$
\mathcal{C}^{*}(L ; P)=\operatorname{Hom}_{U L}\left(\mathcal{C}_{*}(L ; U L), P\right) .
$$

When $Q=U L$ with the action induced by left multiplication then $\mathcal{C}_{*}(L ; U L)=$ $(\bigwedge s L \otimes U L, \partial)$ is a left $\mathcal{C}_{*}(L)$-comodule and a right $U L$-module and both structures are compatible. Moreover, the inclusion $\mathbb{Q} \hookrightarrow \mathcal{C}_{*}(L ; U L)$ is a quasi-isomorphism and $\mathcal{C}_{*}(L ; U L)$ is a semifree $L$-module [12, Proposition 22.3]. Thus,

$$
H\left(\mathcal{C}_{*}(L ; P)\right)=\operatorname{Tor}^{L}(\mathbb{Q}, P) \quad \text { and } \quad H\left(\mathcal{C}^{*}(L ; P)\right)=\operatorname{Ext}_{L}(\mathbb{Q}, P) .
$$


6.2. Cochains with coefficients in the adjoint module $U L_{a}$ and free loop space

Let $(L, \partial)$ be as in 6.1 and consider the adjoint modules $U L_{a}$ and $U L_{a}^{\vee}$ with actions defined by

$$
\begin{array}{ll}
l \cdot x=[l, x] & \text { on } U L_{a}, \\
(f \cdot l)(x)=f([l, x]) & \text { on } U L_{a}^{\vee},
\end{array} \quad l \in L, x \in U L, f \in U L^{\vee} .
$$

Moreover, since $U L$ is a Hopf algebra, for any right (respectively left) differential $L$ modules $(P, \partial)$ (respectively $(Q, \partial))$ the left diagonal L-module $P \otimes Q$ is defined by

$$
x \cdot(a \otimes b)=\sum_{i}(-1)^{\left|x_{i}^{\prime}\right||a|}\left(x_{i} \cdot a\right) \otimes\left(x_{i}^{\prime} \cdot b\right), \quad x \in U L, a \in P, b \in Q,
$$

where $x \mapsto \sum_{i} x_{i} \otimes x_{i}^{\prime}$ denotes the coproduct in $U L$.

Now let $(L, \partial)$ be such that ([12, Lemma 23.1 and (24.b)]) the differential graded algebra $\mathcal{C}^{*}(L)$ is quasi-isomorphic to a minimal model of $M$ :

$$
\mathcal{C}^{*}(L)=(\bigwedge V, d) \simeq \mathfrak{M}_{M}
$$

The differential graded Lie algebra is uniquely defined by this condition. We have $V=$ $s\left(L^{\vee}\right), H(L, \partial)=\pi_{*}(\Omega M)$ and $H_{*}(\Omega M)=H(U L, \partial)$ as Hopf algebras [12, Theorem 21.15]. Moreover, by the Poincaré-Birkhoff-Witt theorem [12, Proposition 21.2], the natural linear isomorphism $\gamma: \bigwedge L \rightarrow U L, x_{1} \wedge \cdots \wedge x_{k} \mapsto(1 / k !) \sum_{\sigma} \varepsilon_{\sigma} x_{\sigma(1)} \cdots x_{\sigma(k)}$, is an isomorphism of graded coalgebras. Therefore we have the following isomorphism of graded algebras:

$$
U L^{\vee} \cong \bigwedge s V
$$

Lemma 4. Considering $U L_{a}$ as a right L-module, the graded linear isomorphisms

$$
\mathcal{C}^{*}\left(L ; U L_{a}^{\vee}\right) \cong \mathcal{C}^{*}\left(L ; U L^{\vee}\right) \cong \mathcal{C}^{*}(L) \otimes U L^{\vee} \cong \mathcal{C}^{*}(L) \otimes \bigwedge s V
$$

define a structure of graded algebra on $\mathcal{C}^{*}\left(L ; U L_{a}^{\vee}\right)$ which is compatible with the differential of $\mathcal{C}^{*}\left(L ; U L_{a}^{\vee}\right)$. Moreover, the natural inclusion

$$
\mathcal{C}^{*}(L) \hookrightarrow \mathcal{C}^{*}\left(L ; U L_{a}^{\vee}\right) \simeq\left(\mathcal{C}^{*}(L) \otimes \wedge s V, d\right), \quad f \mapsto f \otimes 1_{U L^{\vee}},
$$

is a relative Sullivan model of $p_{0}: L M \rightarrow M$.

Proof. Consider the differential graded Lie algebra $L^{S}$ defined as follows:

$$
L_{n}^{S}=L_{n} \oplus \bar{L}_{n}, \quad \bar{L}_{n}=L_{n+1}, \quad d \bar{x}=-\overline{d x}, \quad(-1)^{|a|}[a, \bar{b}]=\overline{[a, b]}, \quad[\bar{a}, \bar{b}]=0 .
$$

Then the inclusion

$$
\mathcal{C}^{*}(L) \hookrightarrow \mathcal{C}^{*}\left(L^{S}\right)=(\bigwedge(V \oplus s V), d)=\left(\mathcal{C}^{*}(L) \otimes \bigwedge s V, d\right) \quad \text { with } \quad d(s v)=-s d(v)
$$

is a relative Sullivan model of $p_{0}: L M \rightarrow M$ (see 4.1, diagram (5)). The rest of the proof follows from the following sequence of isomorphisms of differential graded algebras:

$$
\begin{aligned}
\mathcal{C}^{*}\left(L^{S}\right):=\operatorname{Hom}\left(\mathcal{C}_{*}\left(L ; U L_{a}\right), \mathbb{Q}\right) & \cong \operatorname{Hom}\left(\mathcal{C}_{*}(L ; U L) \otimes_{U L} U L_{a}, \mathbb{Q}\right) \\
& \cong \operatorname{Hom}_{U L}\left(\mathcal{C}_{*}(L ; U L), U L_{a}^{\vee}\right)=: \mathcal{C}^{*}\left(L ; U L_{a}^{\vee}\right) .
\end{aligned}
$$




\subsection{Statement and proof of Theorem $C$}

The multiplication $v: U L \otimes U L \rightarrow U L$ which induces the Pontryagin product on $H_{*}(\Omega M)$ is not a morphism of graded algebras. Nonetheless, $v: U L_{a} \otimes U L_{a} \rightarrow U L_{a}$ is a morphism of $U L$-modules when $U L_{a} \otimes U L_{a}$ is the diagonal left $L$-module. Thus the dual of the product on $U L, v^{\vee}: U L_{a}^{\vee} \rightarrow U L_{a}^{\vee} \otimes U L_{a}^{\vee}$, is a homomorphism of differential graded right $U L$-modules when $U L_{a}^{\vee} \otimes U L_{a}^{\vee}$ denotes the right diagonal module.

Proposition 4. The morphism of complexes

$$
\mathcal{C}^{*}\left(L ; v^{\vee}\right): \mathcal{C}^{*}\left(L, U L_{a}^{\vee}\right) \rightarrow \mathcal{C}^{*}\left(L, U L_{a}^{\vee} \otimes U L_{a}^{\vee}\right) \simeq\left(\mathcal{C}^{*}(L) \otimes(\bigwedge s V)^{\otimes 2}, d\right)
$$

is a homomorphism of commutative differential graded algebras. This is a Sullivan model for the composition of free loops $L M \times_{M} L M \stackrel{\text { Comp }}{\longrightarrow} L M$.

Proof. The contractible chain complexes $\mathcal{C}_{*}(L ; U L)$ and $\mathcal{C}_{*}(U L ; L)$, defined in 6.1 , are differential graded coalgebras via the graded linear isomorphisms $\mathcal{C}_{*}(L ; U L) \cong \mathcal{C}_{*}(L) \otimes$ $U L$ and $\mathcal{C}_{*}(U L ; L) \cong U L \otimes \mathcal{C}_{*}(L)$. Therefore, the coproduct $\mu^{\vee}$ on $\mathcal{C}_{*} L$ induces a quasiisomorphism of differential graded coalgebras

$$
\Phi: \mathcal{C}_{*}(L) \rightarrow \mathcal{C}_{*}(L ; U L) \otimes_{U L} \mathcal{C}_{*}(U L ; L) \cong\left(\mathcal{C}_{*}(L) \otimes U L \otimes \mathcal{C}_{*}(L), \partial\right)
$$

defined by $\Phi(x)=\sum_{i} x_{i} \otimes 1 \otimes x_{i}^{\prime}$ when $\mu^{\vee}(x)=\sum_{i} x_{i} \otimes x_{i}^{\prime}$, and the dual map

$$
\Phi^{\vee}:\left(\mathcal{C}^{*}(L) \otimes U L^{\vee} \otimes \mathcal{C}^{*}(L), d\right):=\left(\mathcal{C}_{*}(L ; U L) \otimes{ }_{U L} \mathcal{C}_{*}(U L ; L)\right)^{\vee} \rightarrow \mathcal{C}^{*}(L)
$$

is a quasi-isomorphism of differential graded algebras. This implies that the natural injection

$$
\begin{gathered}
\mathcal{C}^{*}(L) \otimes \mathcal{C}^{*}(L) \stackrel{\lambda}{\rightarrow}\left(\mathcal{C}^{*}(L) \otimes U L^{\vee} \otimes \mathcal{C}^{*}(L), d\right) \cong\left(\mathcal{C}^{*}(L)^{\otimes 2} \otimes \wedge s V, d\right), \\
x \otimes y \mapsto x \otimes 1 \otimes y,
\end{gathered}
$$

is a relative Sullivan model for the product $\mu: \mathcal{C}^{*}(L) \otimes \mathcal{C}^{*}(L) \rightarrow \mathcal{C}^{*}(L)$ and thus, by diagram $(\mathfrak{M}(4))$ of 4.1 , it is a Sullivan relative model for the path fibration $M^{I} \stackrel{\left(p_{0}, p_{1}\right)}{\longrightarrow} M^{\times 2}$. This fact allows us to follow the construction performed in 4.2 with $\mathfrak{M}_{M^{I}}=\left(\mathcal{C}^{*}(L) \otimes\right.$ $\left.U L^{\vee} \otimes \mathcal{C}^{*}(L), d\right)$ and $\mathfrak{M}_{\sigma^{\prime}}=\Phi^{\vee}$.

First observe that the cochain model of the vertical face on the right in diagram (8),

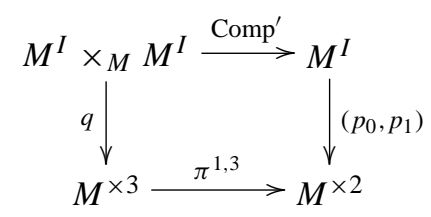


is the pushout diagram

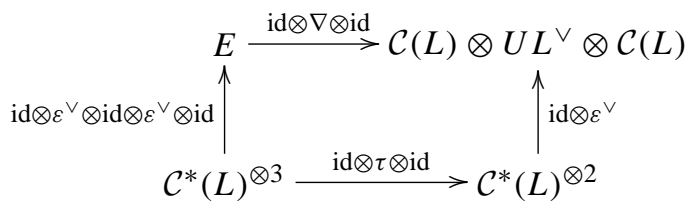

where $\varepsilon: U L \rightarrow \mathbb{Q}$ is the augmentation, $\tau: \mathbb{Q} \hookrightarrow \mathcal{C}(L)$ is the natural inclusion and $\nabla$ is the composition

$$
U L^{\vee} \stackrel{\nu^{\vee}}{\rightarrow}\left(U L^{\vee}\right)^{\otimes 2} \simeq U L^{\vee} \otimes \mathbb{Q} \otimes U L^{\vee} \stackrel{\mathrm{id} \otimes \tau \otimes \mathrm{id}}{\longrightarrow} U L^{\vee} \otimes \mathcal{C}^{*}(L) \otimes U L^{\vee}
$$

Observe that

$$
\begin{aligned}
E & =\mathcal{C}^{*}(L) \otimes U L^{\vee} \otimes \mathcal{C}^{*}(L) \otimes U L^{\vee} \otimes \mathcal{C}^{*}(L) \\
& \simeq\left(\mathcal{C}_{*}(L ; U L) \otimes_{U L} \mathcal{C}_{*}(U L ; L ; U L) \otimes_{U L} \mathcal{C}_{*}(U L ; L)\right)^{\vee}
\end{aligned}
$$

Secondly, we use the machinery developed in (4.2) to translate diagram (8) in terms of Sullivan models. We deduce the explicit model of the map Comp by considering the pushout

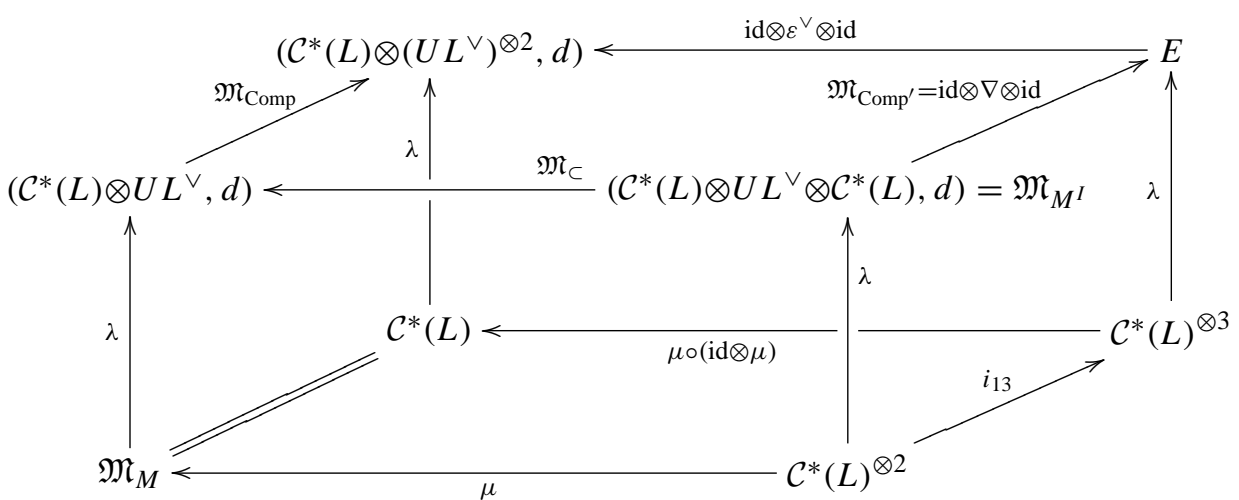

The map $\mathfrak{M}_{\text {Comp }}:\left(\mathcal{C}^{*}(L) \otimes U L^{\vee}, d\right) \rightarrow\left(\mathcal{C}^{*}(L) \otimes\left(U L^{\vee}\right)^{\otimes 2}, d\right)$ is precisely $\mathcal{C}\left(L ; v^{\vee}\right)$.

Remark. Denote by $L_{1}^{S}$ and $L_{2}^{S}$ two copies of $L^{S}$, and $L^{T}=L_{1}^{S} \oplus_{L} L_{2}^{S}=L \oplus \bar{L}_{1} \oplus \bar{L}_{2}$. We denote by $\pi: L^{T} \rightarrow L^{S}$ the projection obtained by mapping identically each $L_{i}^{S}$ to $L^{S}$. We observe that $\mathcal{C}_{*}\left(L^{T}\right)$ is isomorphic to $\mathcal{C}_{*}\left(L ; U L_{a}^{\vee} \otimes U L_{a}^{\vee}\right)$ and that the following 
diagram commutes:

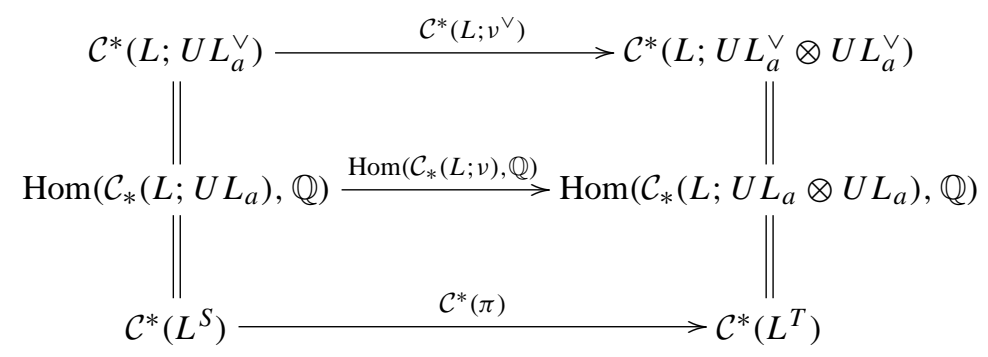

This shows that $\pi: L^{T} \rightarrow L^{S}$ is a "Lie representative" for the composition of free loops $L M \times{ }_{M} L M \rightarrow L M$.

Proposition 4 and Theorem A yield

Theorem C. If $\mathcal{C}^{*}(L)$ is a Sullivan model of $M$ then the dual of the loop product

$$
H^{*}(L M) \stackrel{H^{*}(\mathrm{Comp})}{\longrightarrow} H^{*}\left(L M \times_{M} L M\right) \stackrel{i^{!}}{\rightarrow} H^{*+m}\left(L M^{\times 2}\right)
$$

is induced in homology by the maps

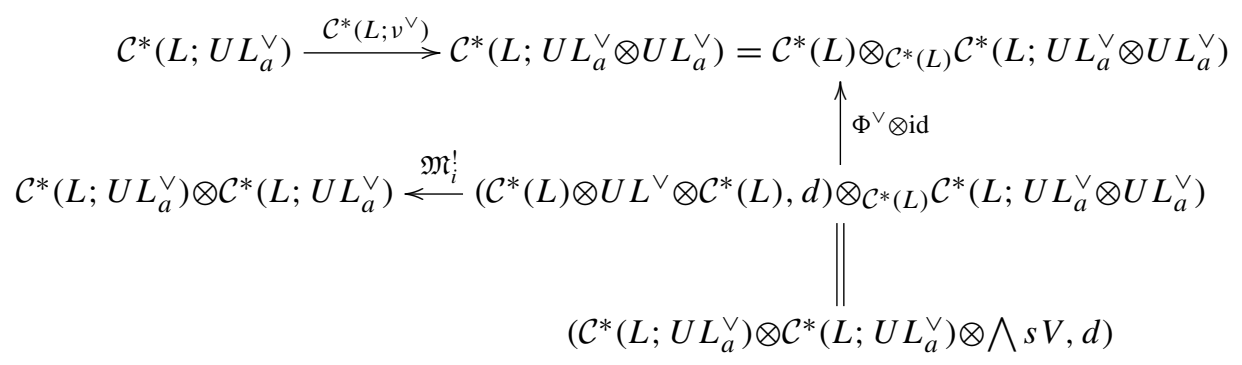

with $\Phi^{\vee}$ defined in (11).

\subsection{Example}

Recall that a coformal space $M$ is a space that admits a Sullivan minimal model with a purely quadratic differential. In this case $\mathcal{C}^{*}(L)=(\bigwedge V, d), L=\pi_{*}(\Omega M) \otimes \mathbb{Q}$ and $H^{*}(\Omega M)=\bigwedge s V$. Thus,

If $M$ is a coformal manifold with minimal model $(\bigwedge V, d)$, then a model for the path composition $L M \times{ }_{M} L M \rightarrow L M$ is given by

$$
\mathrm{id} \otimes v^{\vee}:(\bigwedge V \otimes \bigwedge \bar{V}, d) \rightarrow\left(\bigwedge V \otimes(\bigwedge \bar{V})^{\otimes 2}, d\right),
$$

where $v$ is the Pontryagin product on $H_{*}(\Omega M)$ and $(\bigwedge V \otimes \wedge \bar{V}, d)$ is the model of the free loop space, defined in 4.1 . 
As a particular case consider the 11-dimensional manifold $M$ obtained by taking the pullback of the tangent sphere bundle to $S^{6}$ along the map $f: S^{3} \times S^{3} \rightarrow S^{6}$ that collapses the 3 -skeleton into a point

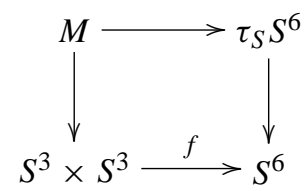

The minimal model of $M$ is

$$
(\bigwedge(x, y, z), d), \quad d x=d y=0, d z=x y, \quad|x|=|y|=3,|z|=5 .
$$

Thus $M$ is a coformal space. A model for the path composition $M^{I} \times{ }_{M} M^{I} \rightarrow M^{I}$ is given by

$$
\begin{aligned}
\varphi:\left(\bigwedge\left(x, y, z, x^{\prime}, y^{\prime}, z^{\prime}, \bar{x}, \bar{y}, \bar{z}\right), d\right) \\
\rightarrow\left(\bigwedge\left(x, y, z, x^{\prime}, y^{\prime}, z^{\prime}, x^{\prime \prime}, y^{\prime \prime}, z^{\prime \prime}, \bar{x}, \bar{y}, \bar{z}, \bar{x}^{\prime}, \bar{y}^{\prime}, \bar{z}^{\prime}\right), d\right),
\end{aligned}
$$

with $d(\bar{x})=x-x^{\prime}, d(\bar{y})=y-y^{\prime}, d(\bar{z})=z-z^{\prime}-\frac{1}{2} \bar{x}\left(y+y^{\prime}\right)+\frac{1}{2}\left(x+x^{\prime}\right) \bar{y}$, $d\left(\bar{x}^{\prime}\right)=x^{\prime}-x^{\prime \prime}, d\left(\bar{y}^{\prime}\right)=y^{\prime}-y^{\prime \prime}, d\left(\bar{z}^{\prime}\right)=z^{\prime}-z^{\prime \prime}-\frac{1}{2} \bar{x}^{\prime}\left(y^{\prime}+y^{\prime \prime}\right)+\frac{1}{2}\left(x^{\prime}+x^{\prime \prime}\right) \bar{y}^{\prime}$, $\varphi(\bar{x})=\bar{x}+\bar{x}^{\prime}, \varphi(\bar{y})=\bar{y}+\bar{y}^{\prime}, \varphi(\bar{z})=\bar{z}+\bar{z}^{\prime}+\frac{1}{2} \bar{x} \bar{y}^{\prime}-\frac{1}{2} \bar{x}^{\prime} \bar{y}$. The induced model for the path composition $L M \times{ }_{M} L M \rightarrow L M$ is then given by

$$
\varphi:(\bigwedge(x, y, z, \bar{x}, \bar{y}, \bar{z}), d) \rightarrow\left(\bigwedge\left(x, y, z, \bar{x}, \bar{y}, \bar{z}, \bar{x}^{\prime}, \bar{y}^{\prime}, \bar{z}^{\prime}\right), d\right),
$$

with $d(\bar{x})=0, d(\bar{y})=0, d(\bar{z})=-\bar{x} y+x \bar{y}, d\left(\bar{x}^{\prime}\right)=0, d\left(\bar{y}^{\prime}\right)=0, d\left(\bar{z}^{\prime}\right)=-\bar{x}^{\prime} y+x \bar{y}^{\prime}$, $\varphi(\bar{x})=\bar{x}+\bar{x}^{\prime}, \varphi(\bar{y})=\bar{y}+\bar{y}^{\prime}, \varphi(\bar{z})=\bar{z}+\bar{z}^{\prime}+\frac{1}{2} \bar{x} \bar{y}^{\prime}-\frac{1}{2} \bar{x}^{\prime} \bar{y}$.

\section{Dual of the loop product in terms of chains on a differential graded Lie algebra}

\subsection{The cap-homomorphism}

Let $(L, \partial)$ be a differential graded Lie algebra, $N$ be a differential graded $L$-module and $\mathcal{C}_{*}(L ; N), \mathcal{C}^{*}(L ; N)$ be as in 6.1. Let $c=\sum_{i} s x_{i_{1}} \wedge \cdots \wedge s x_{i_{q}} \in \mathcal{C}_{q}(L)$ be a cycle of degree $m$. We define the cap product by $c$ :

$$
\operatorname{cap}_{c}: \mathcal{C}^{q-r}(L ; N)=\operatorname{Hom}_{U L}\left(\mathcal{C}_{q-r}(L ; U L), N\right) \rightarrow \mathcal{C}_{r}(L ; N), \quad f \mapsto f \cap c,
$$

where

$$
\begin{aligned}
f \cap c= & (-1)^{m} \sum_{i} \sum_{\sigma \in \Sigma_{q}}(-1)^{|f| \cdot \mid s x_{\sigma\left(i_{1}\right)} \wedge \cdots \wedge s x_{\sigma\left(i_{r}\right) \mid}} \varepsilon_{\sigma} \\
& \cdot s x_{\sigma\left(i_{1}\right)} \wedge \cdots \wedge s x_{\sigma\left(i_{r}\right)} \otimes f\left(s x_{\sigma\left(i_{r+1}\right)} \wedge \cdots \wedge s x_{\sigma\left(i_{q}\right)}\right) .
\end{aligned}
$$

A standard computation proves:

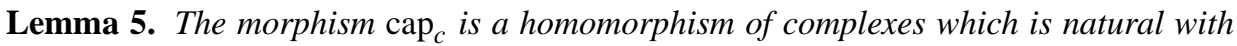
respect to homomorphisms of differential graded L-modules. 


\subsection{Statement of Theorem D}

Since the dual of the multiplication $v^{\vee}: U L_{a}^{\vee} \rightarrow U L_{a}^{\vee} \otimes U L_{a}^{\vee}$ is a morphism of $U L$ modules, Lemma 5 implies:

Proposition 5. The following diagram commutes:

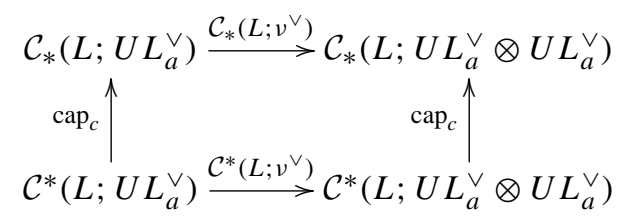

The next result, whose proof is postponed to Subsection 7.3, furnishes a representative of the Gysin map $i^{!}: H^{*}\left(L M \otimes_{M} L M\right) \rightarrow H^{*+m}\left(L M^{\times 2}\right)$ at the level of chains.

Let $(L, \partial)$ be such that $\mathcal{C}^{*}(L) \simeq \mathfrak{M}_{M}$. The algebra $H^{*}\left(\mathcal{C}^{*}(L)\right)=H^{*}(M)$ is a Poincaré duality algebra with a fundamental class $[M] \in H_{m}(M)$. We denote by $u$ a cycle in $\mathcal{C}_{*}(L)$ representing $[M]$. Then an easy spectral sequence argument shows that $\operatorname{cap}_{u}: \mathcal{C}^{*}(L ; N) \rightarrow \mathcal{C}_{*}(L ; N)$ is a quasi-isomorphism.

Proposition 6. With the notation of 6.2, the following diagram commutes in homology:

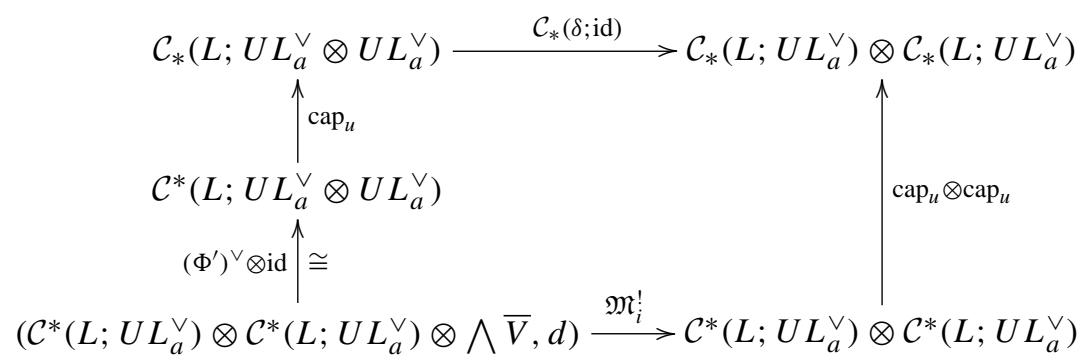

Here $\delta: L \rightarrow L \oplus L, x \mapsto(x, x)$.

It follows directly from Propositions 5 and 6 that the coproduct

$$
\mathcal{C}_{*}\left(L ; U L_{a}^{\vee}\right) \stackrel{\mathcal{C}_{*}\left(L ; v^{\vee}\right)}{\longrightarrow} \mathcal{C}_{*}\left(L ; U L_{a}^{\vee} \otimes U L_{a}^{\vee}\right) \stackrel{\mathcal{C}_{*}(\delta ; \mathrm{id})}{\longrightarrow} \mathcal{C}_{*}\left(L ; U L_{a}^{\vee}\right) \otimes \mathcal{C}_{*}\left(L ; U L_{a}^{\vee}\right)
$$

induces on $H_{*}(L M)$ a coproduct

$$
H_{*}(L M) \rightarrow H_{*}(L M) \otimes H_{*}(L M)
$$

which is identified via the linear map $H\left(\operatorname{cap}_{u}\right)$ with the dual of the loop product

$$
H^{*}(L M) \rightarrow\left(H^{*}(L M) \otimes H^{*}(L M)\right)^{*+m} .
$$

Now, observe that this coproduct on $\mathcal{C}_{*}\left(L ; U L_{a}^{\vee}\right)$ coincides with the coproduct obtained by the tensor product of the coalgebra $\mathcal{C}^{*}(L)$ with the coalgebra $\left(U L^{\vee}, v^{\vee}\right)$. Thus we have proved the following result: 
Theorem D. The homology of the differential graded coalgebra $\mathcal{C}_{*}\left(L ; U L_{a}^{\vee}\right)$ is isomorphic, via the isomorphism induces by cap $_{u}$, to the coalgebra $\mathbb{H}^{*}(L M):=H^{*-m}(L M)$ equipped with the dual of the loop product.

It is worthwhile to observe here that there exists an isomorphism of differential graded algebras between the dual algebra $\operatorname{Hom}\left(\mathcal{C}_{*}\left(L ; U L_{a}^{\vee}\right), \mathbb{Q}\right)$ and the differential graded algebra $\mathcal{C}^{*}\left(L ; U L_{a}\right)$. The structure of graded algebra on $\mathcal{C}^{*}\left(L ; U L_{a}\right)$ is defined by the tensor product of the two graded algebras: $\mathcal{C}^{*}(L) \otimes(U L, v)$.

\subsection{Proof of Proposition 6}

We will use the following general result about modules over differential graded algebras.

Lemma 6. Let $R$ be a differential graded algebra, $S$ be a left semifree $R$-module, and let $f, g: N \rightarrow P$ be homomorphisms of right $R$-modules. If $H(f)=H(g)$ then the maps $f \otimes 1, g \otimes 1: N \otimes_{R}(R \otimes V) \rightarrow P \otimes_{R}(R \otimes V)$ induce the same map in homology.

Proof. Recall from 2.4 that $S=\sum_{n \geq 0} R \otimes W(n)$ with $d W(n) \subset R \otimes \sum_{k<n} W(k)$ and where each $W(n)$ is a graded vector space. The subcomplexes $F^{p}\left(N \otimes_{R} S\right)=\sum_{k \leq p} N \otimes$ $(R \otimes W(k))$ (respectively $\left.F^{p}\left(P \otimes_{R} S\right)=\sum_{k<p} P \otimes(R \otimes W(k))\right)$ form a filtration that induces a spectral sequence converging to $H\left(N \otimes_{R} S\right)$ (respectively $H\left(P \otimes_{R} S\right)$ ). Since $E_{1}(f \otimes \mathrm{id})=H(f) \otimes \mathrm{id}=H(g) \otimes \mathrm{id}=E_{1}(g \otimes \mathrm{id})$ we deduce that $H(f \otimes \mathrm{id})=$ $H(g \otimes \mathrm{id})$.

The next result specifies the relation between the map $\mathcal{C}_{*}(\delta)$ and the Gysin map of the diagonal embedding (see 4.3).

Lemma 7. With the previous notations the following diagram is commutative:

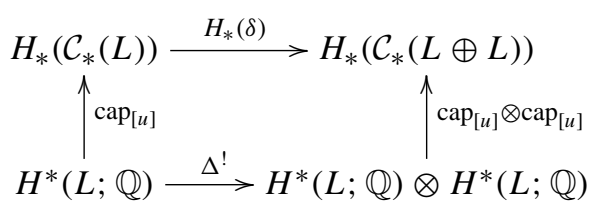

Proof. By direct computation using definitions introduced in 4.3.

End of proof of Proposition 6. We define $R=\mathcal{C}^{*}(L) \otimes \mathcal{C}^{*}(L)$. We remark that $R \otimes V$ $=\mathcal{C}^{*}\left(L ; U L_{a}^{\vee}\right) \otimes \mathcal{C}^{*}\left(L ; U L_{a}^{\vee}\right)$ is a semifree $R$-module. Note also that the diagram in Proposition 6 is the tensor product of a diagram of $R$-modules by $R \otimes V$ over $R$. By Lemma 7, this diagram commutes in homology when $V=\mathbb{Q}$. It then commutes in homology by Lemma 6 . This completes the proof. 


\section{Loop product and Gerstenhaber product}

\subsection{Bar construction and chains of a differential graded Lie algebra}

Let $(A, d)$ be a differential graded supplemented algebra, $A=\mathbb{Q} \oplus \bar{A}$, and $(P, d)$ (resp. $(Q, d))$ be a right (respectively left) differential graded $A$-module. The (normalized) twosided bar construction $\mathbb{B}(P ; A ; Q)$ is defined as follows:

$$
\mathbb{B}_{k}(P ; A ; Q)=P \otimes T^{k} s \bar{A} \otimes Q
$$

where $T^{k} V=V^{\otimes k}$. A generic element is written $m\left[a_{1}\left|a_{2}\right| \cdots \mid a_{k}\right] n$ with degree $|m|+$ $|n|+\sum_{i=1}^{k}\left|s a_{i}\right|$. The differential $d=d_{0}+d_{1}$ is defined by

$$
\begin{aligned}
& \mathbb{B}_{k}(P ; A ; Q) \stackrel{d_{0}}{\rightarrow} \mathbb{B}_{k}(P ; A ; Q), \quad \mathbb{B}_{k}(P ; A ; Q) \stackrel{d_{1}}{\rightarrow} \mathbb{B}_{k-1}(P ; A ; Q), \\
& d_{0}\left(p\left[a_{1}\left|a_{2}\right| \cdots \mid a_{k}\right] q\right)= d(p)\left[a_{1}\left|a_{2}\right| \cdots \mid a_{k}\right] q \\
&-\sum_{i=1}^{k}(-1)^{|p|+\epsilon_{i}} p\left[a_{1}\left|a_{2}\right| \cdots\left|d\left(a_{i}\right)\right| \cdots \mid a_{k}\right] q \\
&+(-1)^{|p|+\epsilon_{k+1}} p\left[a_{1}\left|a_{2}\right| \cdots \mid a_{k}\right] d(q), \\
& d_{1}\left(p\left[a_{1}\left|a_{2}\right| \cdots \mid a_{k}\right] q\right)=(-1)^{|p|} p a_{1}\left[a_{2}|\cdots| a_{k}\right] q \\
&+\sum_{i=2}^{k}(-1)^{|p|+\epsilon_{i}} p\left[a_{1}\left|a_{2}\right| \cdots\left|a_{i-1} a_{i}\right| \cdots \mid a_{k}\right] q \\
&-(-1)^{|p|+\epsilon_{k}} p\left[a_{1}\left|a_{2}\right| \cdots \mid a_{k-1}\right] a_{k} q
\end{aligned}
$$

where $\epsilon_{i}=\sum_{j<i}\left|s a_{j}\right|$. The (normalized) bar construction on $A$ is the differential graded coalgebra $\mathbb{B}(A):=\mathbb{B}(k ; A ; k)=\left(T^{c}(s \bar{A}), d\right)$. The relation between the bar construction on $U L$ and the chains on $L$ is given by the following result:

Proposition 7 ([12, Proposition 22.7]). The canonical homomorphism

$$
\begin{aligned}
& \mathcal{C}_{*}(P ; L ; Q) \stackrel{\Psi}{\rightarrow} \mathbb{B}(P ; U L ; Q), \\
& p \otimes s x_{1} \wedge \cdots \wedge s x_{k} \otimes q \mapsto p \otimes\left(\sum_{\sigma \in \Sigma_{k}} \varepsilon_{\sigma}\left[x_{\sigma(1)}|\cdots| x_{\sigma(k)}\right]\right) \otimes q,
\end{aligned}
$$

is a quasi-isomorphism. In particular:

a) if $P=N=U L$ is the canonical L-bimodule then $\Phi: \mathcal{C}_{*}(U L ; L ; U L) \rightarrow$ $\mathbb{B}(U L ; U L ; U L)$ is also a quasi-isomorphism of differential graded $U L$-bimodules,

b) if $P=Q=\mathbb{Q}$ is the trivial L-module then $\psi: \mathcal{C}_{*}(L) \rightarrow \mathbb{B}(U L)$ is a quasi-isomorphism of differential graded coalgebras. 


\subsection{The Hochschild complex and chains with coefficients in an adjoint module}

Let $A=\mathbb{Q} \oplus \bar{A}$ be a supplemented algebra, $A^{\text {op }}$ be the opposite algebra and $A^{e}=A \otimes$ $A^{\text {op }}$ be the enveloping algebra of $A$; thus $A^{e}$-modules are $A$-bimodules. The Hochschild cochain complex of $A$ with coefficients in the $A$-bimodule $P$ is the differential module

$$
C C^{*}(A ; P)=\left(\operatorname{Hom}\left(T^{c}(s \bar{A}), P\right), D_{0}+D_{1}\right) \cong \operatorname{Hom}_{A^{e}}(\mathbb{B}(A ; A ; A), P)
$$

where the differential $D_{0}+D_{1}$ of $f \in \operatorname{Hom}\left(T^{c}(s \bar{A}), P\right)$ is defined by

$$
\begin{aligned}
D_{0}(f)\left(\left[a_{1}\left|a_{2}\right| \cdots \mid a_{k}\right]\right)= & d_{M}\left(f\left(\left[a_{1}\left|a_{2}\right| \cdots \mid a_{k}\right]\right)\right) \\
& -\sum_{i=1}^{k}(-1)^{|f|+\epsilon_{i}} f\left(\left[a_{1}|\cdots| d_{A} a_{i}|\cdots| a_{k}\right]\right), \\
D_{1}(f)\left(\left[a_{1}\left|a_{2}\right| \cdots \mid a_{k}\right]\right)= & (-1)^{\left|s a_{1}\right||f|} a_{1} f\left(\left[a_{2}|\cdots| a_{k}\right]\right) \\
& -\sum_{i=2}^{k}(-1)^{|f|+\epsilon_{i}} f\left(\left[a_{1}|\cdots| a_{i-1} a_{i}|\cdots| a_{k}\right]\right) \\
& +(-1)^{|f|+\epsilon_{k}} f\left(\left[a_{1}\left|a_{2}\right| \cdots \mid a_{k-1}\right]\right) a_{k} .
\end{aligned}
$$

The Hochschild cohomology of A with coefficients in $M$ is

$$
H H^{*}(A ; M)=H\left(C C^{*}(A ; M)\right)=H\left(\left(\operatorname{Hom}\left(T^{c}(A), M\right), D_{0}+D_{1}\right)\right) .
$$

The product defined on $\operatorname{Hom}\left(T^{c}(s \overline{U L}), U L\right)$ (see 2.2) commutes with the differential $D_{0}+D_{1}$. This is the Gerstenhaber product [15]. The next result specifies how the Gerstenhaber product on $C C^{*}(U L ; U L)$ and the differential graded coalgebra $\mathcal{C}^{*}\left(L ; U L_{a}^{\vee}\right)$, as considered in Theorem D, are related. The proof of this result relies upon the "inverse process" of Cartan-Eilenberg [5].

Proposition 8. Let L be a connected differential graded Lie algebra. Then there exists a canonical isomorphism of graded algebras

$$
H H^{*}(U L ; U L) \cong \operatorname{Hom}\left(H_{*}\left(\mathcal{C}_{*}\left(L ; U L_{a}^{\vee}\right)\right), \mathbb{Q}\right) .
$$

Proof. We form the commutative diagram

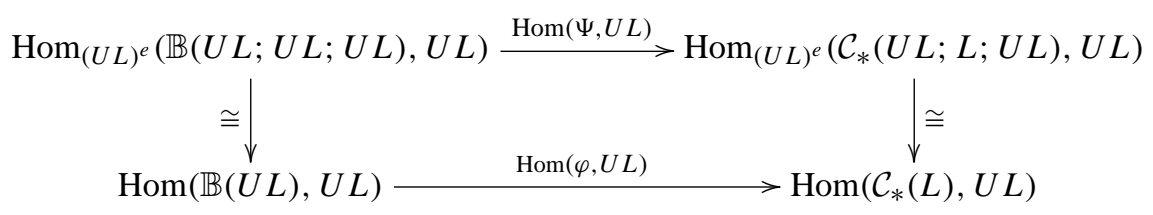

where the vertical maps are the canonical isomorphisms of graded vector spaces. These isomorphisms define the graded algebra structure on

$\operatorname{Hom}_{(U L)^{e}}(\mathbb{B}(U L ; U L ; U L), U L)$ and $\operatorname{Hom}_{(U L)^{e}}\left(\mathcal{C}_{*}(U L ; L ; U L), U L\right)$. 
Therefore they can be viewed as isomorphisms of graded algebras (but not as isomorphisms of differential graded algebras). Since $\operatorname{Hom}(\psi, U L)$ is a homomorphism of graded algebras the map $\operatorname{Hom}(\Psi, U L)$ is a quasi-isomorphism of differential graded algebras.

Considering the commutative diagram

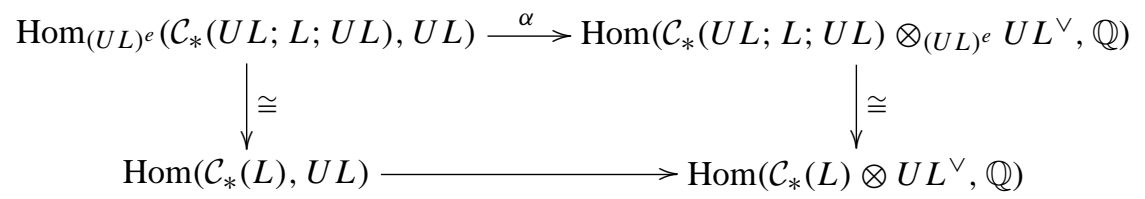

one proves, in the same manner, that the canonical isomorphism $\alpha$ is an isomorphism of differential graded algebras.

Finally, a straightforward computation shows that the canonical linear isomorphism

$$
\mathcal{C}_{*}\left(L ; U L_{a}^{\vee}\right) \stackrel{\beta}{\rightarrow} \mathcal{C}_{*}(U L ; L ; U L) \otimes_{(U L)^{e}} U L^{\vee}
$$

is an isomorphism of chain complexes. From the commutative diagram

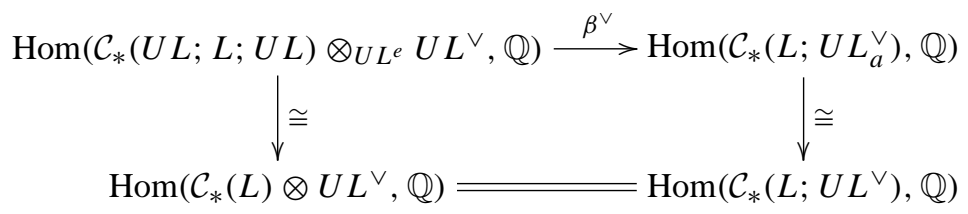

we deduce that $\beta^{\vee}$ is an isomorphism of differential graded algebras.

The composition $\beta^{\vee} \circ \alpha \circ \operatorname{Hom}(\Psi, U L)$ induces a canonical isomorphism of graded algebras

$$
H H^{*}(U L ; U L) \cong \operatorname{Hom}\left(H_{*}\left(\mathcal{C}_{*}\left(L ; U L_{a}^{\vee}\right)\right), \mathbb{Q}\right)
$$

\subsection{Statement and proof of Theorem $E$}

Assume that $\mathcal{C}^{*}(L)$ is a Sullivan model of $M$. Using the natural isomorphism of graded algebras $([13])$

$$
H H^{*}(U L ; U L) \cong H H^{*}\left(\mathcal{C}^{*}(L) ; \mathcal{C}^{*}(L)\right),
$$

and the existence of quasi-isomorphisms of differential graded algebras, $\mathcal{C}^{*}(L) \stackrel{\cong}{\cong}$ $C^{*}(M)$, we deduce from Proposition 8 and Theorem D:

Theorem E. Let $M$ be a simply connected closed oriented manifold. There exists a natural isomorphism of graded algebras

$$
J: \mathbb{H}_{*}(L M) \stackrel{\cong}{\rightarrow} H H^{*}\left(C^{*}(M) ; C^{*}(M)\right) .
$$




\section{I-homomorphism and Hochschild homology}

\subsection{A convenient definition of the I-homomorphism}

For each subspace $Z \hookrightarrow M$, we denote by $L_{Z} M$ the space of loops in $L M$ with base point in $Z$. We thus have the commutative pullback diagram

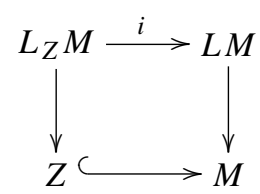

Denote now by $D$ a closed disk around the base point $x_{0}$. Since $D$ is contractible, $L_{D} M$ is homotopy equivalent to $D \times \Omega M$. The $I$-homomorphism $I: H_{*}(L M) \rightarrow$ $H_{*-m}(\Omega M)$ is the composition

$$
\begin{aligned}
\tilde{H}_{*}(L M) \rightarrow H_{*}\left(L M, L_{M-x_{0}} M\right) \stackrel{\text { Excision }}{\longrightarrow} H_{*}\left(L_{D} M, L_{S^{m-1}} M\right) \\
\\
\cong H_{m}\left(D, S^{m-1}\right) \otimes H_{*-m}(\Omega M) .
\end{aligned}
$$

Therefore the $I$-homomorphism is precisely the Gysin map of the embedding $\Omega M \hookrightarrow$ $L M$.

Let $(\bigwedge V \otimes \bigwedge s V, d)$ be a relative Sullivan model for the free loop space (4.1, diagram (5)), and let $\omega \in(\bigwedge V)^{m}$ be a cocycle representing the fundamental class. Then the direct sum $\mathcal{J}=(\bigwedge V)^{>m} \oplus S$, where $(\bigwedge V)^{m}=\mathbb{Q} \cdot \omega \oplus S$, is an acyclic differential ideal in $\bigwedge V$; the quotient map $q:(\bigwedge V, d) \rightarrow(A, d)=(\bigwedge V / \mathcal{J}, d)$ is a quasi-isomorphism. We form the tensor product

$$
(A \otimes \wedge s V, d):=(A, d) \otimes \wedge V(\bigwedge V \otimes \wedge s V, d) .
$$

Lemma 7. The natural injection $i:(\bigwedge s V, 0) \rightarrow(A \otimes \bigwedge s V, d)$ defined by $i(a)=$ $(-1)^{m} \omega \otimes$ a is a morphism of complexes of degree $m$ inducing in cohomology the dual of the I-homomorphism, $I^{\vee}: H^{*}(\Omega M) \rightarrow H^{*+m}(L M)$.

Proof. A model for the injection $L_{M-x_{0}} M \hookrightarrow L M$ is given by the quotient map $q$ : $(A \otimes \wedge s V, d) \rightarrow(A /(\omega) \otimes \bigwedge s V, d)$. Therefore a model for the cochain complex $\mathcal{C}^{*}\left(L M, L_{M-x_{0}} M\right)$ is given by $\operatorname{Ker} q=(\mathbb{Q} \cdot \omega \otimes \bigwedge \bar{V}, 0)$. This implies that a model for the composition

$$
H^{*}(\Omega M) \stackrel{\cong}{\rightrightarrows} H^{*+m}\left(L M, L_{M-x_{0}} M\right) \rightarrow H^{*+m}(L M)
$$

is given by the composition $\bigwedge s V \stackrel{a \mapsto(-1)^{m} \omega \otimes a}{\longrightarrow} \mathbb{Q} \cdot \omega \otimes \bigwedge \bar{V} \rightarrow H^{*}(A \otimes \bigwedge s V, d)$. 


\subsection{Statement and proof of Theorem $F$}

Theorem F. Let $J$ be as in Theorem E. There exists a canonical isomorphism of graded algebras, $\bar{J}$, making the diagram

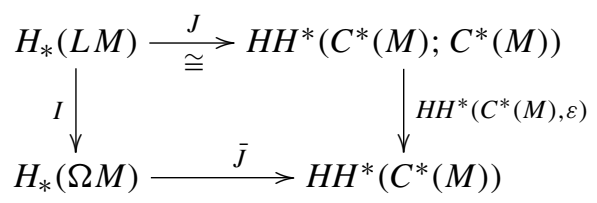

commutative, where $\varepsilon: C^{*} M \rightarrow \mathbb{Q}$ denotes the usual augmentation.

Proof. Let $(L, \partial)$ be such that $\mathcal{C}^{*}(L)=\mathfrak{M}_{M}$. The algebra $\mathcal{C}^{*}\left(L ; U L_{a}^{\vee}\right)=\mathcal{C}^{*}(L) \otimes U L^{\vee}$ is then a relative Sullivan model for the free loop space $L M$, and we can apply Lemma 6 (see 7.3) to this model to have a model for the restriction morphism. Now, since the projection $q: \mathcal{C}^{*}\left(L ; U L_{a}^{\vee}\right) \rightarrow A \otimes U L_{a}^{\vee}$ is a quasi-isomorphism, $q$ admits a lifting $q^{\prime}: U L^{\vee} \rightarrow \mathcal{C}^{*}\left(L ; U L_{a}^{\vee}\right)$.

Let $u$ be a cycle in $\mathcal{C}(L)$ representing the orientation class $[M]$ such that $\langle\omega,[M]\rangle=1$. We obtain a commutative diagram

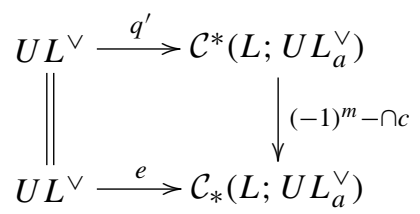

where $e(a)=1 \otimes a$. The dual diagram yields in homology a diagram of graded algebras whose vertical maps are isomorphisms:

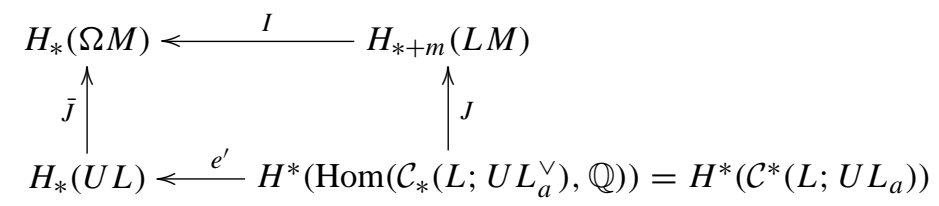

Note that the two $\mathcal{C}_{*}(L)$-bicomodules $\mathcal{C}_{*}(L ; U L) \otimes_{U L} \mathcal{C}_{*}(U L ; L)$ and $\mathcal{C}_{*}(L)$ are quasiisomorphic. Therefore we have by duality a quasi-isomorphism of $\mathcal{C}^{*}(L)$-bimodules $\mathcal{C}^{*}(L) \otimes U L^{\vee} \otimes \mathcal{C}^{*}(L)=\left(\mathcal{C}_{*}(L ; U L) \otimes_{U L} \mathcal{C}_{*}(U L ; L)\right)^{\vee} \rightarrow \mathcal{C}^{*}(L)$. We deduce the isomorphism of differential graded vector spaces

$$
\begin{aligned}
C C^{*}(U L ; U L) & =\operatorname{Hom}_{(U L)^{e}}\left(\mathcal{C}_{*}(U L ; L ; U L), U L\right), \\
C C^{*}\left(C^{*}(L) ; C^{*}(L)\right) & =\operatorname{Hom}_{\mathcal{C}^{*}(L)^{e}}\left(\left(\mathcal{C}_{*}(L ; U L) \otimes_{U L} \mathcal{C}_{*}(U L ; L)\right)^{\vee}, \mathcal{C}^{*}(L)\right) .
\end{aligned}
$$

The isomorphisms

$$
\begin{aligned}
\operatorname{Hom}_{(U L)^{e}}\left(\mathcal{C}_{*}(U L ; L ; U L), U L\right) & \cong \operatorname{Hom}\left(\mathcal{C}_{*}(L), U L\right), \\
\operatorname{Hom}_{\mathcal{C}^{*}(L)^{e}}\left(\left(\mathcal{C}_{*}(L ; U L) \otimes_{U L} \mathcal{C}_{*}(U L ; L)\right)^{\vee}, \mathcal{C}^{*}(L)\right) & \cong \operatorname{Hom}\left(U L^{\vee}, \mathcal{C}^{*}(L)\right)
\end{aligned}
$$


induce differentials on the right hand side terms. Now a simple computation (see for instance [13]) shows that the map $D$ that associates to a map in $\operatorname{Hom}\left(C_{*}(L), U L\right)$ the dual map in $\operatorname{Hom}\left(U L^{\vee}, \mathcal{C}^{*}(L)\right)$ is an isomorphism of complexes. This induces the following commutative diagram of complexes:

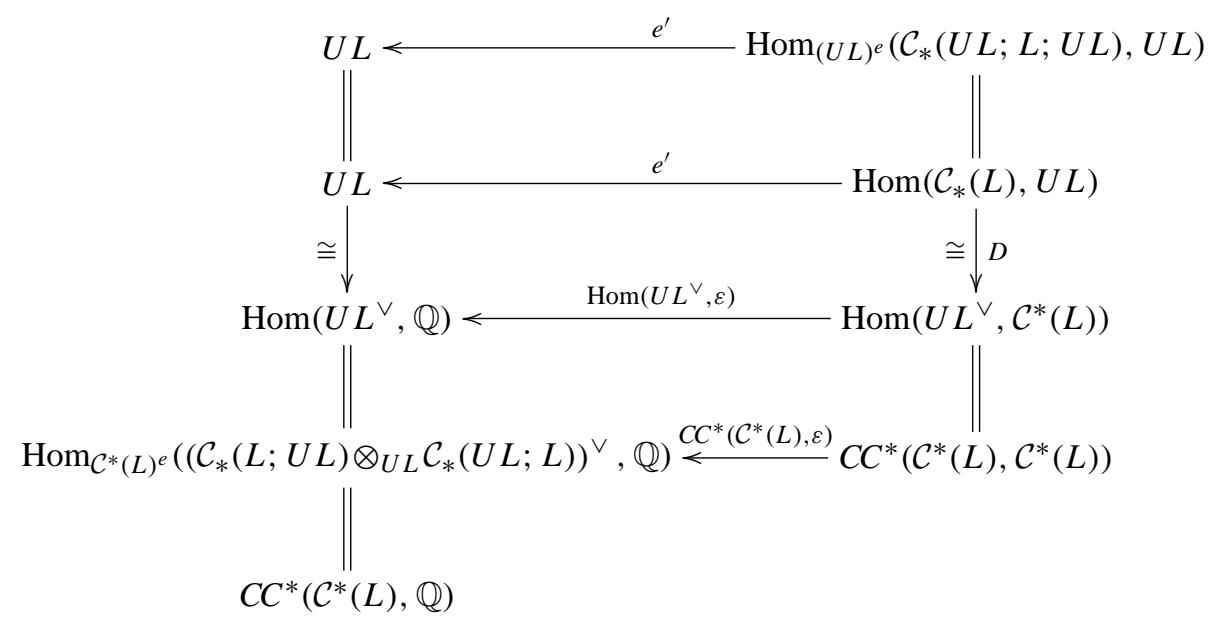

where $\varepsilon: \mathcal{C}^{*}(L) \rightarrow \mathbb{Q}$ denotes the canonical augmentation. The result follows now directly from the induced diagram in cohomology, combined with diagram $(*)$.

\section{References}

[1] Baker, A., Õze, C.: Complex cobordism of Hilbert manifolds with some applications to flag varieties of loop groups. In: Geometry and Topology (Aarhus, 1998), Comtemp. Math. 258, Amer. Math. Soc., Providence, RI, 1-19 (2000) Zbl 0979.57013 MR 1778093

[2] Bredon, G. E.: Topology and Geometry. Grad. Texts in Math. 139, Springer (1993) Zbl 0791.55001 MR 1224675

[3] Brylinski, J.-L.: Loop Spaces, Characterisic Classes and Geometric Quantization. Progr. Math. 107, Birkhäuser Boston, Boston, MA (1993) Zbl 0823.55002 MR 1197353

[4] Burghelea, D., Vigué-Poirrier, M.: A model for cyclic homology and algebraic K-theory. J. Differential Geom. 22, 243-253 (1985) Zbl 0595.55009 MR 0834279

[5] Cartan, H., Eilenberg, S.: Homological Algebra. Princeton Univ. Press (1999) Zbl 0933.18001 MR 1731415

[6] Chas, M., Sullivan, D.: String topology. Preprint CUNY, Nov. 1999, math.GT/9911159, to appear

[7] Chataur, D.: A bordism approach to string topology. Int. Math. Res. Not. 2005, no. 46, 28292875 Zbl pre02246597 MR 2180465

[8] Cohen, R. L., Godin, V.: A polarized view of string topology. In: Topology, Geometry and Quantum Field Theory, London Math. Soc. Lecture Note Ser. 308, Cambridge Univ. Press, Cambridge, 127-154 (2004) Zbl 1095.55006 MR 2079373

[9] Cohen, R. L., Jones, J. D. S., Yan, J.: The loop homology algebra of spheres and projective spaces. In: Categorical Decomposition Techniques in Algebraic Topology, Progr. Math. 215, Birkhäuser, 77-92 (2004) Zbl 1054.55006 MR 2039760 
[10] Deligne, P., Griffiths, J., Morgan, J., Sullivan, D.: Real homotopy theory of Kähler manifolds. Invent. Math. 29, 245-274 (1975) Zbl 0312.55011 MR 0382702

[11] Félix, Y., Halperin, S., Thomas, J.-C.: Gorenstein spaces. Adv. Math. 71, 92-112 (1988) Zbl 0659.57011 MR 0960364

[12] Félix, Y., Halperin, S., Thomas, J.-C.: Rational Homotopy Theory. Grad. Texts in Math. 205, Springer (2001) Zbl 0961.55002 MR 1802847

[13] Félix, Y., Menichi, L., Thomas, J.-C.: Gerstenhaber duality in Hochschild cohomology. J. Pure Appl. Algebra 199, 43-59 (2005) Zbl 1076.55003 MR 2134291

[14] Félix, Y., Thomas, J.-C., Vigué-Poirrier, M.: The Hochschild cohomology of a closed manifold. Inst. Hautes Études Sci. Publ. Math. 99, 235-252 (2004) Zbl 1060.57019 MR 2075886

[15] Gerstenhaber, M.: The cohomology structure of an associative ring. Ann. of Math. 78, 267288 (1963) Zbl 0131.27302 MR 0161898

[16] Halperin, S., Stasheff, J. D.: Obstructions to homotopy equivalences. Adv. Math. 32, 233-279 (1979) Zbl 0408.55009 MR 0539532

[17] Hatcher, A.: Algebraic Topology. Cambridge Univ. Press (2002) Zbl 1044.55001 MR 1867354

[18] Lang, S.: Differential and Riemannian Manifolds. 3rd ed., Grad. Texts in Math. 160, Springer, New York (1995) Zbl 0824.58003 MR 1335233

[19] Merkulov, S. A.: De Rham model for string topology, Int. Math. Res. Not. 2004, no. 55, 2955-2987 Zbl 1066.55008 MR 2099178

[20] Milnor, J., Stasheff, J. D.: Characteristic Classes. Ann. of Math. Stud. 76, Princeton Univ. Press (1974) Zbl 0298.57008 MR 0440554

[21] Sullivan, D.: Infinitesimal computations in topology. Inst. Hautes Études Sci. Publ. Math. 47, 269-331 (1977) Zbl 0374.57002 MR 0646078

[22] Sullivan, D.: Open and closed string field theory interpreted in classical algebraic topology. In: Topology, Geometry and Quantum Field Theory, London Math. Soc. Lecture Note Ser. 308, Cambridge Univ. Press, Cambridge, 344-357 (2004) MR 2079379

[23] Vigué-Poirrier, M.: Homologie cyclique des espaces formels. J. Pure Appl. Algebra 91, 347 354 (1994) Zbl 0802.55011 MR 1255938 